\title{
High-Energy Neutrino Production in Clusters of Galaxies
}

\author{
Saqib Hussain, ${ }^{1}{ }^{\star}$ Rafael Alves Batista, ${ }^{2} \dagger$ Elisabete M. de Gouveia Dal Pino, ${ }^{3} \ddagger$ \\ and Klaus Dolag, \\ ${ }_{1,3}$ Institute of Astronomy, Geophysics and Atmospheric Sciences (IAG), University of São Paulo (USP), São Paulo, Brazil \\ ${ }^{2}$ Radboud University Nijmegen, Department of Astrophysics/IMAPP, 6500 GL Nijmegen, The Netherlands \\ ${ }^{4}$ University Observatory Munich, Scheinerstr. 1, 81679 Munchen, Germay \\ ${ }^{5}$ Max Planck Institute for Astrophysics, Karl-Schwarzschild-Str 1, 85741 Garching, Germany
}

Accepted XXX. Received YYY; in original form ZZZ

\begin{abstract}
Clusters of galaxies can potentially produce cosmic rays (CRs) up to very-high energies via large-scale shocks and turbulent acceleration. Due to their unique magnetic-field configuration, CRs with energy $\leq 10^{17} \mathrm{eV}$ can be trapped within these structures over cosmological time scales, and generate secondary particles, including neutrinos and gamma rays, through interactions with the background gas and photons. In this work we compute the contribution from clusters of galaxies to the diffuse neutrino background. We employ three-dimensional cosmological magnetohydrodynamical simulations of structure formation to model the turbulent intergalactic medium. We use the distribution of clusters within this cosmological volume to extract the properties of this population, including mass, magnetic field, temperature, and density. We propagate CRs in this environment using multidimensional Monte Carlo simulations across different redshifts (from $z \sim 5$ to $z=0$ ), considering all relevant photohadronic, photonuclear, and hadronuclear interaction processes. We find that, for CRs injected with a spectral index $\alpha=1.5-2.7$ and cutoff energy $E_{\max }=10^{16}-5 \times 10^{17} \mathrm{eV}$, clusters contribute to a sizeable fraction to the diffuse flux observed by the IceCube Neutrino Observatory, but most of the contribution comes from clusters with $M \gtrsim 10^{14} M_{\odot}$ and redshift $z \lesssim 0.3$. If we include the cosmological evolution of the CR sources, this flux can be even higher.
\end{abstract}

Key words: galaxies: clusters: intracluster medium, neutrinos, magnetic fields

\section{INTRODUCTION}

The IceCube Neutrino Observatory reported evidence of an isotropic distribution of neutrinos with $\sim \mathrm{PeV}$ energies (Aartsen et al. 2017, 2020). Their origin is not known yet, but the isotropy of the distribution suggests that they are predominantly of extragalactic origin. They might come from various types of sources, such as galaxy clusters (Murase et al. 2013; Hussain et al. 2019), starbursts galaxies, galaxy mergers, AGNs (Murase et al. 2013; Kashiyama \& Mészáros 2014; Anchordoqui et al. 2014; Khiali \& de Gouveia Dal Pino 2016; Fang \& Murase 2018), supernova remnants (Chakraborty \& Izaguirre 2015; Senno et al. 2015), gamma-ray bursts (Hümmer et al. 2012; Liu \& Wang 2013). Since neutrinos can reach the Earth without being deflected by magnetic fields or attenuated due to any sort of interaction, they can help to unveil the sources of ultra-high-energy cosmic rays (UHECRs) that produce them.

Their origin and that of the diffuse gamma-ray emission are among the major mysteries in astroparticle physics. The fact that the observed energy fluxes of UHECRs, high-energy neutrinos, and gamma rays are all comparable suggests that these messengers may have some connection with each other (Ahlers \& Halzen 2018; Alves Batista

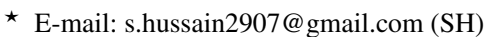

$\dagger$ E-mail: r.batista@astro.ru.nl

†-mail: dalpino@iag.usp.br
}

et al. 2019a; Ackermann et al. 2019). The three fluxes could, in principle, be explained by a single class of sources (Fang \& Murase 2018), like starburst galaxies or galaxy clusters (e.g., Murase et al. 2008; Kotera et al. 2009; Alves Batista et al. 2019a, for reviews ).

Clusters of galaxies form in the universe possibly through violent processes, like accretion and merging of smaller structures into larger ones (Voit 2005). These processes release large amounts of energy, of the order of the gravitational binding energy of the clusters $(\sim$ $10^{61}-10^{64} \mathrm{erg}$ ). Part of this energy is depleted via shock waves and turbulence through the intracluster medium (ICM), which accelerate CRs to relativistic energies. These can be also re-accelerated by similar processes in more diffuse regions of the ICM, including relics, halos, filaments, and cluster mergers (e.g., Brunetti \& Jones 2014; Brunetti \& Vazza 2020, for reviews). Furthermore, clusters of galaxies are attractive candidates for UHECR production due to their extended sizes $(\simeq \mathrm{Mpc})$ and suitable magnetic field strength $(\sim 1 \mu \mathrm{G})$ (e.g., Fang \& Murase 2018; Kim et al. 2019). Those with energies $E>7 \times 10^{18} \mathrm{eV}$ have most likely an extragalactic origin (e.g., Aab et al. 2018; Alves Batista et al. 2019b), and those with $E \lesssim 10^{17} \mathrm{eV}$ are believed to have Galactic origin (see e.g., Blasi 2013; Amato \& Blasi 2018), although the exact transition between galactic and extragalactic CRs is not clear yet (see e. g., Aloisio et al. 2012; Parizot 2014; Giacinti et al. 2015; Thoudam et al. 2016; Kachelriess 2019).

CRs with $E \lesssim 10^{17} \mathrm{eV}$ can be confined within clusters for a time 
comparable to the age of the universe (e.g. Hussain et al. 2019). This confinement makes clusters efficient sites for the production of secondary particles including, electron-positron pairs, neutrinos and gamma rays due to their interaction with the thermal protons and photon fields (e.g. Berezinsky et al. 1997; Rordorf et al. 2004; Kotera et al. 2009). Non-thermal radio to gamma-ray and neutrino observations are, therefore, the most direct ways of constraining the properties of CRs in clusters (Berezinsky et al. 1997; Wolfe \& Melia 2008; Yoast-Hull et al. 2013; Zandanel et al. 2015). Conversely, the diffuse flux of gamma rays and neutrinos depend on the energy budget of CR protons in the ICM. Clusters also naturally can introduce a spectral softening due to the fast escape of high-energy CRs from the magnetized environment which might explain the second knee that appears around $\sim 10^{17} \mathrm{eV}$, in the CR spectrum (Apel et al. 2013).

To calculate the fluxes of CRs and secondary particles from clusters, there are many analytical and semi-analytical works (Berezinsky et al. 1997; Wolfe \& Melia 2008; Murase et al. 2013), but in most of the approaches, the ICM model is overly simplified by assuming, for instance, uniform magnetic field and gas distribution. There are more realistic numerical approaches in Rordorf et al. (2004) and Kotera et al. (2009) exploring the three-dimensional (3D) magnetic fields of clusters. More recently, Fang \& Olinto (2016) estimated the flux of neutrinos from these objects assuming an injected CR spectrum $\propto E^{-1.5}$, an isothermal gas distribution, a radial profile for the total matter (baryonic and dark) density profile, and a Kolmogorov turbulent magnetic field with coherence length $\sim 100 \mathrm{kpc}$. They found these estimates to be comparable to IceCube measurements. Here we revisit these analyses by employing a more rigorous numerical approach. We take into account the non-uniformity of the gas density and magnetic field distributions in clusters, as obtained from MHD simulations. We consider additional factors such as the location of $\mathrm{CR}$ sources within a given cluster, and the obvious mass dependence of the physical properties of clusters. This last consideration is important because massive clusters $\left(\gtrsim 10^{15} M_{\odot}\right)$ are much less common than lower-mass ones $\left(\lesssim 10^{13} M_{\odot}\right)$. Consequently, clusters that can confine CRs of energy above PeV for longer are probably more relevant for detection of high-energy neutrinos.

Our main goal is to derive the contribution of clusters to the diffuse flux of high-energy neutrinos. To this end, we follow the propagation and cascading of CRs and their by-products in the cosmological background simulations by Dolag et al. (2005). We use the Monte Carlo code CRPropa (Alves Batista et al. 2016) that accounts for all relevant photohadronic, photonuclear, and hadronuclear interaction processes. Ultimately, we obtain the $\mathrm{CR}$ and neutrino fluxes that emerge from the clusters.

This paper is organized as follows: in section 2 we describe the numerical setup for both the cosmological background simulations and for CR propagation through this environment; in section 3 we characterize the 3D-MHD simulations and present our results for the fluxes of CRs and neutrinos; in section 4 we discuss our results; finally, in section 5 we draw our conclusions.

\section{NUMERICAL METHOD}

\subsection{Background MHD Simulation}

To study the propagation of CRs in the ICM we consider the large scale cosmological 3D-MHD simulations performed by Dolag et al. (2005), who employed the Lagrangian smoothed particle hydrodynamics (SPH) code GADGET (Springel et al. 2001; Springel 2005). These simulations capture the essential features of the mass, tem-
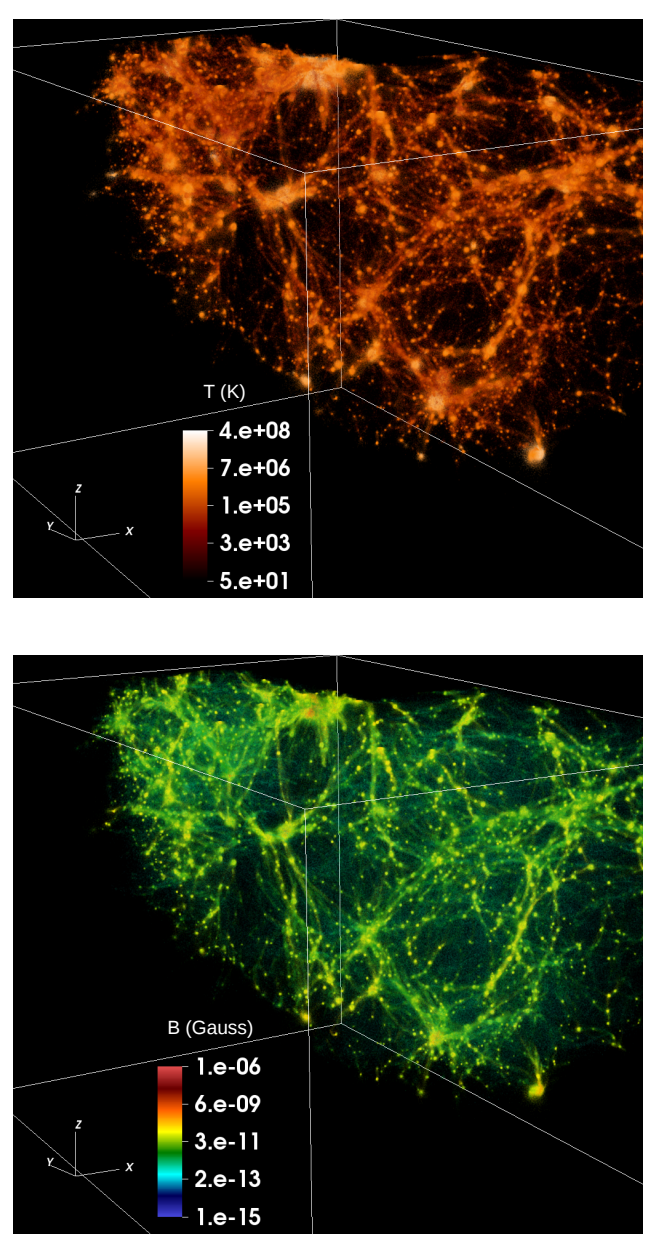

Figure 1. This figure shows the temperature (upper panel) and magnetic field (lower panel) for one of the eight regions of our background 3D-MHD cosmological simulation at redshift $z=0.01$, with dimension $240 \mathrm{Mpc}^{3}$, performed by Dolag et al. (2005).

perature, density, and magnetic field distributions in galaxy clusters, filaments and voids.

We consider here seven snapshots of these simulations with redshifts $z=0.01 ; 0.05 ; 0.2 ; 0.5 ; 0.9 ; 1.5 ; 5.0$, each having the same volume $(240 \mathrm{Mpc})^{3}$. We have divided the domain of each snapshot into eight regions. Fig. 1 shows the temperature and magnetic-field distributions for one of the regions, at redshift $z=0.01$.

The filaments in Fig. 1 are populated with galaxy clusters and have dimensions $\sim 50 \mathrm{Mpc}^{3}$, while the voids have dimensions of the same order, which are compatible with observations (e.g., Govoni et al. 2019; Gouin et al. 2020). In this simulation, the comoving intensity of the seed magnetic field was chosen to be $B=2 \times 10^{-12} \mathrm{G}$, which leads to a quite reasonable match with the field strength observed in different clusters of galaxies today. Feedback and star formation were not included in these cosmological simulations. The background cosmological parameters assumed are $h \equiv H_{0} /\left(100 \mathrm{~km} \mathrm{~s}^{-1} \mathrm{Mpc}^{-1}\right)=0.7$, $\Omega_{m}=0.3, \Omega_{\Lambda}=0.7$, and the baryonic fraction $\Omega_{b} / \Omega_{m}=14 \%$. 


\subsection{Simulation Setup for Cosmic Rays}

The simulations described in the previous section provide the background magnetic field, gas density and temperature distributions of the ICM. In order to study the CR propagation in this environment, we employ the CRPropa 3 code (Alves Batista et al. 2016), with stochastic differential equations (Merten et al. 2017).

In these simulations, we assume that CRs are composed only by protons. We consider all relevant interactions during their propagation including photohadronic, photonuclear, and hadronuclear processes, namely photopion production, photodisintegration, nuclear decay, proton-proton ( $\mathrm{pp}$ ) interactions, and adiabatic losses due to the expansion of the universe. The cosmic microwave background radiation $(\mathrm{CMB})$ and the extragalactic background light $(\mathrm{EBL})$ are two essential ingredients, but other contributions comes from the hot gas component of the ICM, of temperatures between $\sim 10^{6}-10^{8} \mathrm{~K}$, that produces bremsstrahlung radiation (Rybicki \& Lightman 2008) and serves as target for pp-interactions. This is calculated in section 3.1 .

\subsubsection{Cosmic-ray Propagation}

To investigate the flux of different particle species and the change of their energy spectrum, we use the Parker transport equation, which is a simplified version of the Fokker-Planck equation. It gives a good description of the transport of CRs for an isotropic distribution in the diffuse regime. It is given by:

$$
\frac{\partial n}{\partial t}+\vec{u} \cdot \nabla n=\nabla \cdot(\hat{\kappa} \nabla n)+\frac{1}{p^{2}} \frac{\partial}{\partial p}\left(p^{2} \kappa p p \frac{\partial n}{\partial p}\right)+\frac{1}{3}(\nabla \vec{u}) \frac{\partial n}{\partial \ln p}+S(\vec{x}, p, t) .
$$

Here $\vec{u}$ is the advection speed, $\hat{\kappa}$ is the spatial diffusion tensor, $p$ is the absolute momentum, $\kappa_{p p}$ is the diffusion coefficient of momentum used to describe the reacceleration, $\mathrm{n}$ is the particle density, $\vec{x}$ gives position and $S(\vec{x}, p, t)$ is the source of CRs (distribution of CRs at the source).

Propagation of CRs can be diffusive or semi-diffusive, depending on the Larmor radius $\left(r_{\mathrm{L}}=1.08 E_{15} / B_{\mu \mathrm{G}} \mathrm{pc}\right)$ of the particles and the magnetic field of the ICM. The diffusive regime corresponds to $r_{\mathrm{L}} \ll$ $R_{\text {cluster, }}$, and the semi-diffusive is for $r_{\mathrm{L}} \gtrsim R_{\text {cluster, }}$, wherein $R_{\text {cluster }}$ is the radius of the cluster, typically $\sim 1 \mathrm{Mpc}$. Because $B \sim \mu \mathrm{G}$, for the energy range of interest $\left(10^{14}-10^{19} \mathrm{eV}\right), r_{\mathrm{L}} \ll R_{\text {cluster }}$, so we are in the diffusive regime. CRs in this energy range would be confined completely by the magnetic field of the clusters for a time longer than the Hubble time $\left(t_{\mathrm{H}} \sim 14 \mathrm{Gyr}\right)$ (e.g. Fang \& Murase 2018). For instance, a CR with energy $\sim 10^{17} \mathrm{eV}$ in a cluster of mass $\sim 10^{14} M_{\odot}$ with central magnetic field strength $\sim 10^{-6} \mu \mathrm{G}$ has $r_{\mathrm{L}} \sim 0.1 \mathrm{kpc}$ much smaller than the size of the cluster $(\sim 2 \mathrm{Mpc})$ and the trajectory length of this CRs inside the cluster is $\sim 10^{3} \mathrm{Mpc}$. The confinement time for this CR can be calculated as $t_{\text {con }} \sim 1000 \mathrm{Mpc} / c \sim t_{\mathrm{H}}$ (e.g. Hussain et al. 2019). Hence, CRs with energy $E>10^{17} \mathrm{eV}$ have more chances to escape the magnetized cluster environment. The flux of CRs that can escape a cluster depends on its mass and magnetic-field profile, with the latter directly correlated with the density distribution, being larger in denser regions.

\section{RESULTS}

\subsection{Cosmological Background}

Our background simulation includes seven snapshots in the redshift range $0.01<z<5$.0. We have identified clusters in the densest regions of the isocontour maps of the whole volume, in each snapshot (see Fig. 1). We then selected five clusters with distinct masses ranging from $10^{12}$ to $10^{16} M_{\odot}$, which we assumed to be representative of all the clusters in the corresponding snapshot. Finally, we injected CRs in each of these clusters to study their propagation and production of secondary particles. As an example, Fig. 2 illustrates relevant properties for two of these clusters with masses $\sim 10^{14} M_{\odot}$ (left panel) and $\sim 10^{15} M_{\odot}$ (right panel) at redshift $z=0.01$. To estimate the total mass of a cluster from the simulations, we integrated the baryonic and dark matter densities within a volume of $2 \mathrm{Mpc}$, assuming an approximate spherical volume. We note that this specific evaluation is not much affected by the deviations from spherical symmetry that we detect in Fig. 2.

To illustrate general average properties of the simulated clusters, we converted the Cartesian into spherical coordinates and divided the cluster in 10 concentric spherical shells of different radii $\left(R_{\text {shell }}\right)$. Starting from the center of the cluster, the shells were first divided in intervals of $100 \mathrm{kpc}$, then between $300 \mathrm{kpc}$ and $1500 \mathrm{kpc}$, they were divided in intervals of $200 \mathrm{kpc}$, and the last shell in the outskirts was taken between $1500 \mathrm{kpc}<r<2000 \mathrm{kpc}$.

Fig. 3 depicts volume-averaged profiles of different quantities as a function of the radial distance for a cluster of mass $\sim 10^{15} M_{\odot}$ at four different redshifts. The overdensity in Fig. 3 (bottom-right panel) is defined as $\Delta=\rho(r) / \rho_{\text {bary }}$, where $\rho(r)$ is the total density at a given point and $\rho_{\text {bary }}$ is the mean baryonic density, $\rho_{\text {bary }}=\Omega_{\text {bary }} \times \rho_{\text {crit }}$, $\rho_{\text {crit }}=3 H^{2} / 8 \pi \mathrm{G}$. We see that, in general, these radial profiles are very similar across the cosmological time, except for the temperature that varies non-linearly with time by about four orders of magnitude in the inner regions of the cluster. Fig. 4 shows profiles for the temperature, gas density, magnetic field and overdensity for a cluster of mass $\sim 10^{15} M_{\odot}$, as a function of the azimuthal $(\phi)$ angle for different latitudes $(\theta)$, within a radial distance of $R=300 \mathrm{kpc}$, at a redshift $z=0.01$. We see that there are substantial variations in the angular distributions of all the quantities. These variations characterize a deviation from spherical symmetry that may affect the emission pattern of the CRs and consequently secondary gamma rays and neutrinos.

We also found that the magnetic field strength of a cluster depends on its mass: the heavier the cluster, the stronger the average magnetic field is, due to the larger extension of denser regions (see middle column of Fig. 2 and Fig. 5). Inside all clusters, magnetic fields vary in the range $10^{-8}<B / \mathrm{G}<10^{-5}$ (see also Dolag et al. 2005; Ferrari et al. 2008; Xu et al. 2009; Brunetti \& Jones 2014; Brunetti et al. 2017; Brunetti \& Vazza 2020).

In the upper panel of Fig. 6, we compare the radial density profile of our simulated cluster of mass $10^{15} M_{\odot}$ with the model used by Fang \& Olinto (2016). We see that both profiles look similar up to $\sim 10^{3} \mathrm{kpc}$. Above this scale, the density distribution of our simulated clusters decays much faster than the assumed distribution in Fang \& Olinto (2016).

To estimate the total flux of CRs and neutrinos, we need to evaluate the total number of clusters in our background simulations as a function of their mass, at different redshifts. From the entire simulated volume, $(240 \mathrm{Mpc})^{3}$, we selected 20 sub-samples of $(20 \mathrm{Mpc})^{3}$ from different regions, as representative of the whole background. We then calculated the average number of clusters per mass interval in each of these sub-samples $\left(d N_{\text {clusters, avg }} / d M\right)$, between $10^{12} M_{\odot}$ and $10^{16} M_{\odot}$. To obtain the total number of clusters per mas interval we multiplied this quantity by the number of intervals $N=(240 \mathrm{Mpc})^{3} /(20 \mathrm{Mpc})^{3}$ in which the whole volume was divided. So, the total number of clusters per 

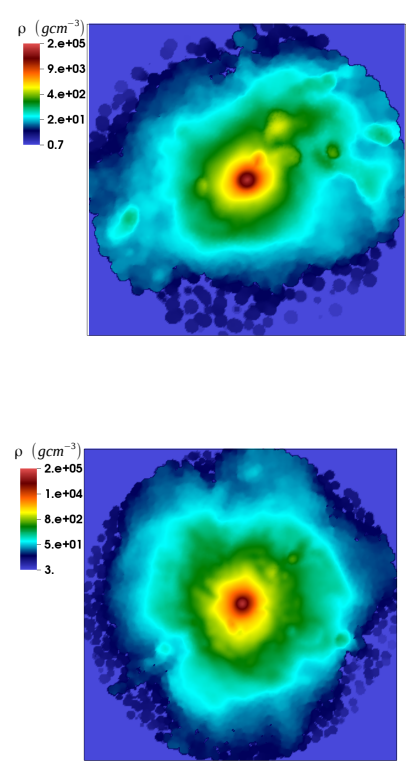
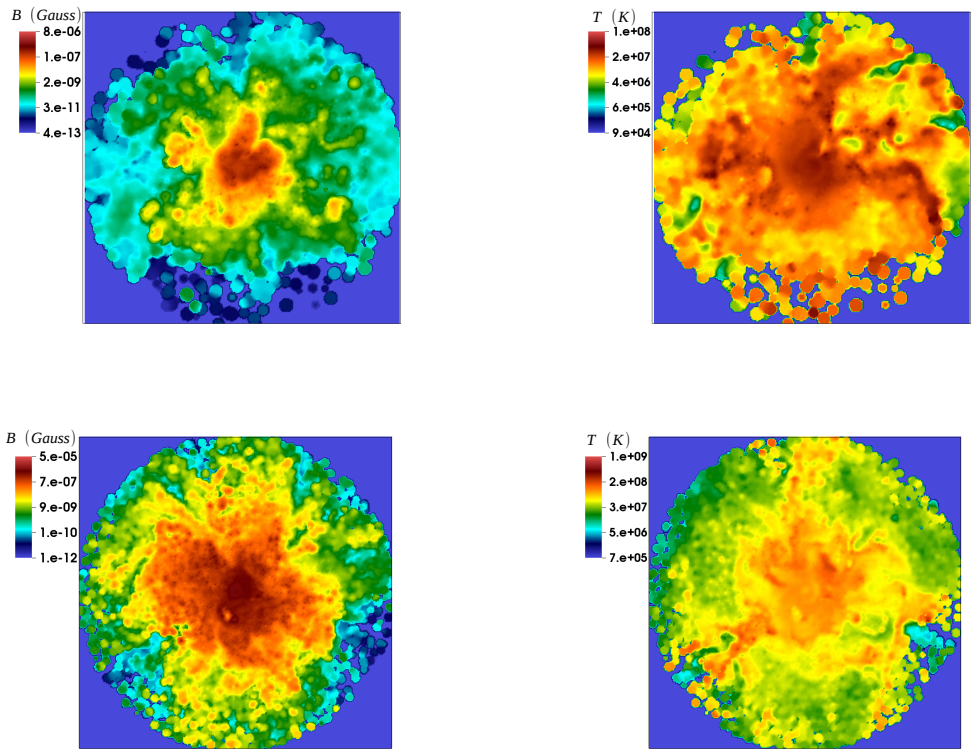

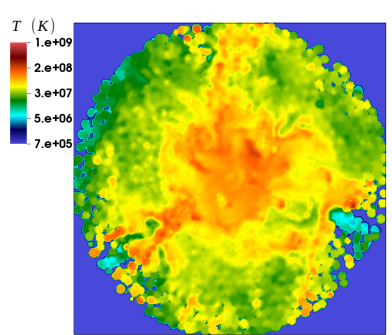

Figure 2. Maps of gas density (left column), magnetic field (middle column), temperature (right column) of two clusters of masses $\sim 10^{14} M_{\odot}($ upper panels) and $\sim 10^{15} M_{\odot}$ (bottom panels), at redshift $z=0.01$.
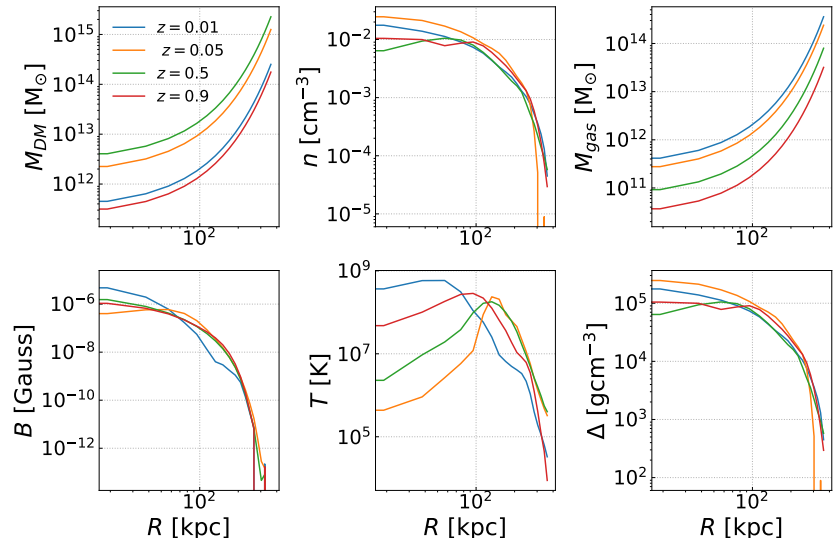

Figure 3. Volume-averaged profiles as a function of the radial distance from the center for a cluster of mass $M \sim 10^{15} M_{\odot}$, at four different redshifts. The quantities shown are: dark-matter mass (top left); gas number density (top center); gas mass (top right); magnetic field (bottom left); temperature (bottom-center) and overdensity (bottom right).

mass interval was calculated as $\left(d N_{\text {clusters, avg }} / d M\right) \times N$. Since we have seven redshifts in our cosmological background simulations, $z=0.01,0.05,0.2,0.5,0.9,1.5,5.0$, we then have repeated the calculation above for each snapshot to obtain the number of clusters per mass interval at different redshifts. This is shown in the lower panel of Fig. 6 for different redshifts.

To calculate the photon field of the ICM, we assume that the clusters are filled with photons from Bremsstrahlung radiation of the hot, rarefied ICM gas (see Figs. 1 to 4). For typical temperatures and densities, we can further assume an optically thin gas. Taking a photon density $\left(n_{\mathrm{ph}}\right)$ distribution with approximately spherical symmetric
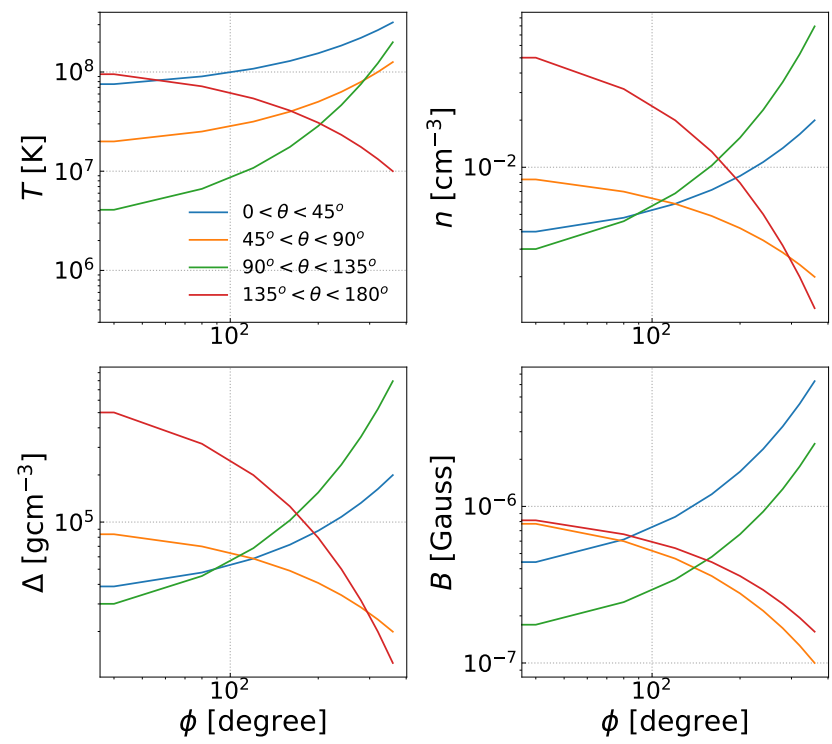

Figure 4. Volume-averaged profiles as a function of the azimuthal $(\phi)$ angle for different latitudes $(\theta)$, within a radial distance $R=300 \mathrm{kpc}$ from the center, for a cluster of mass $M \sim 10^{15} M_{\odot}$. From top left to bottom right clockwise, temperature, gas number density, overdensity and magnetic field.

within the cluster, we have the following relations for an optically thin gas (Rybicki \& Lightman 2008):

$\frac{d n_{\mathrm{ph}}}{d \epsilon}=\frac{4 \pi I_{v}}{c h \epsilon}, \quad I_{v}=R_{\text {shell }} J_{v}^{\mathrm{ff}}$.

where $I_{v}$ is the specific intensity of the emission, $c$ is the speed of light, $h$ is the Planck constant, $\epsilon$ is the photon energy, $R_{\text {shell }}$ is the radius of concentric spherical shells, and $J_{v}^{\mathrm{ff}}$ is related with the 

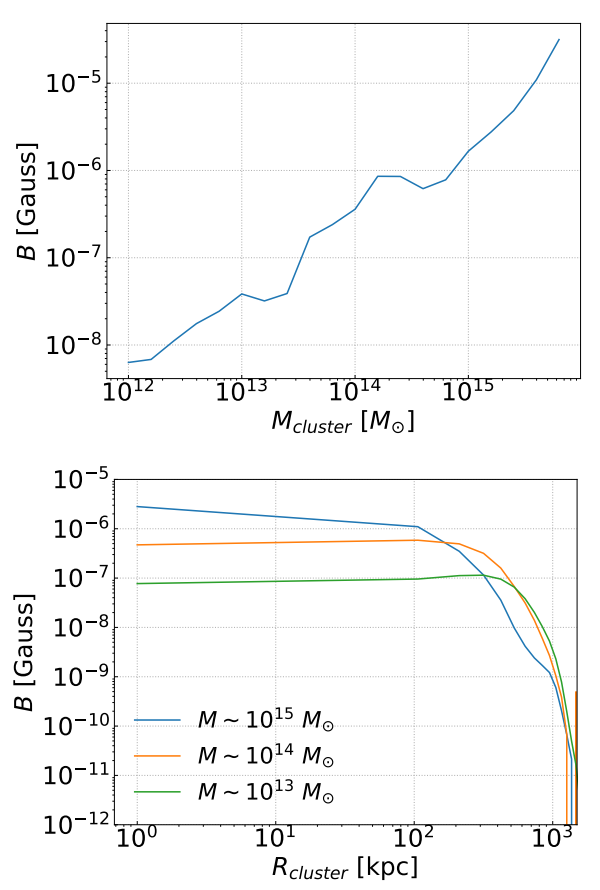

Figure 5. Upper panel shows the whole volume-averaged value of the magnetic field as a function of the cluster mass. Lower panel compares the volumeaveraged magnetic field as a function of the radial distance for clusters of different masses.
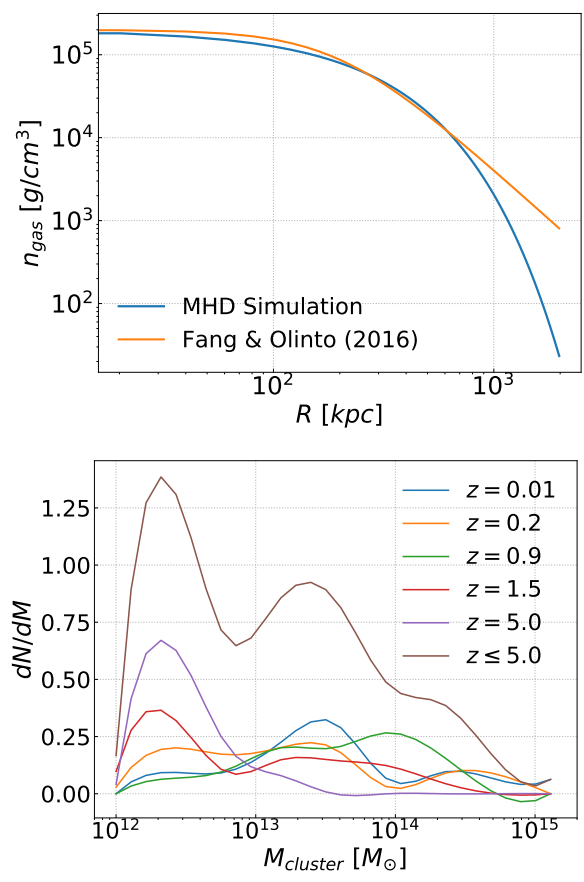

Figure 6. Comparison of the density profile of a cluster of mass $10^{15} \boldsymbol{M}_{\odot}$, from our simulation with the model used by Fang \& Olinto (2016), given in the upper panel. The lower panel shows the number of clusters per mass interval in our background simulation for different redshifts.

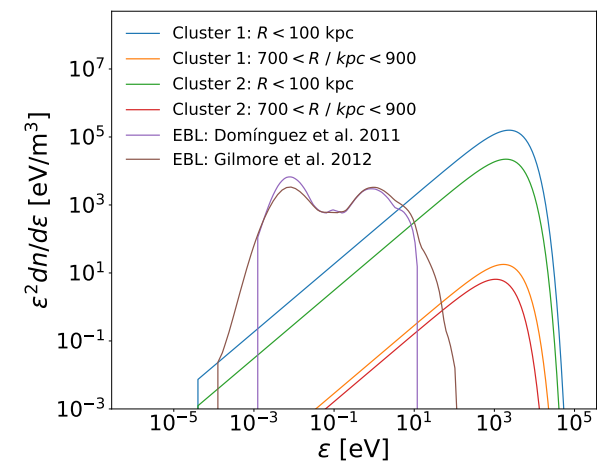

Figure 7. Comparison of EBL with the Bremsstrahlung radiation of the ICM as a function of the photon energy. The Bremsstrahlung is calculated for two clusters at different radial distance intervals. Cluster 1 has mass $10^{15} M_{\odot}$, and Cluster $2,10^{14} M_{\odot}$.

Bremsstrahlung emission coefficient:

$4 \pi J_{v}^{\mathrm{ff}}=\epsilon_{v}^{\mathrm{ff}}(v, n, T)=6.8 \times 10^{-38} Z^{2} n_{e} n_{i} T^{-1 / 2} e^{-h v / k_{B} T}$,

which is given in units of erg $\mathrm{cm}^{-3} \mathrm{~s}^{-1} \mathrm{~Hz}^{-1}$.

In Fig. 7 we compare the radiation fields for two EBL models (Gilmore et al. 2012; Dominguez et al. 2011) with the Bremsstrahlung photon fields of two clusters of masses $\sim 10^{15} M_{\odot}$ (cluster 1) and $\sim 10^{14} M_{\odot}$ (cluster 2$)$. For both clusters, we calculated the internal photon field at the center $(R<100 \mathrm{kpc})$ and for the (700 $<R / \mathrm{kpc}<900)$. It can be seen that the Bremsstrahlung photon field is dominant at X-rays, but only near the center of the clusters, while the EBL dominates at infrared and optical wavelengths mainly.

The interaction rates of CRs with the Bremsstrahlung photon fields in each shell were also calculated (see Fig. 8 and appendix A) and implemented in CRPropa. We note that though the assumption of spherical symmetry for evaluating the Bremsstrahlung radiation and its interaction rate with CRs seems to be in contradiction with the results of Fig. 4, our computation of these quantities in CRPropa have revealed no significant contribution of the Bremsstrhalung photons to neutrinos production. Indeed, the upper panel of Fig. 8 indicates that the $\lambda$ for these interactions is larger than the Hubble horizon. Thus deviations from spherical symmetry for this photon field will not be relevant in this study.

We have also implemented the proton-proton (pp) interactions using the spatial dependent density field extracted directly from the background cosmological simulations, using the same procedure described by Rodríguez-Ramírez et al. (2019). We further notice that, for the computation of the CR fluxes, the magnetic field distribution has been also extracted directly from the background simulations, without considering any kind of space symmetry.

\subsection{Mean free paths for different CR interactions}

CRPropa 3 employs a Monte Carlo method for particle propagation and previously loaded tables of the interaction rates in order to calculate the interaction of CRs with photons along their trajectories. We implemented the spatially-dependent interaction rates into the code, based on the gas and photon density distributions for the clusters of different masses. The mean free paths $(\lambda)$ for the different interactions of CRs are described in appendix A.

The values of $\lambda$ for all the interactions of CRs with the background photon fields and the gas, are plotted in the upper panel of Fig. 8. For photopion production, we compare $\lambda$ due to interactions with the 
photon fields (i.e., the Bremsstrahlung radiation, red solid line) of a cluster of mass $10^{15} M_{\odot}$ with the EBL (red dotted line) and the CMB (red dashed line). For the Bremsstrahlung radiation, we considered only the photons within a sphere of radius $100 \mathrm{kpc}$ around the center of the cluster (i.e., the densest region, which is shown in Figs. 2 \& 4). High-energy CR interactions with CMB photons is a wellunderstood process that limits the distance from which CRs can reach Earth leading to the GZK cutoff. The upper panel of Fig. 8 shows that $\lambda$ for this interaction is much smaller than that for the EBL and Bremsstrahlung. So, CR interactions with $\mathrm{CMB}$ photons dominate at energies $E \gtrsim 10^{17} \mathrm{eV}$. We also see that $\lambda$ for Bremsstrahlung is greater than the size of the universe $\left(\sim 10^{6} \mathrm{Mpc}\right)$, and for EBL, it is $\sim 10^{3} \mathrm{Mpc}$. The $\lambda$ for pp-interactions (green line) is much less than the Hubble horizon. Therefore, this kind of interaction is more likely to occur than photopion production specially at energies $<10^{17} \mathrm{eV}$. Upper panel of Fig. 8 also shows that we can neglect the $\mathrm{CR}$ interactions with the local Bremsstrahlung photon field, as well as the interaction of high-energy gamma rays with the local gas of the ICM (yellow) in photopion production.

The lower panel of Fig. 8 shows the distribution of the trajectory lengths (total distance travelled by a CR inside the cluster up to the observation time), for different energy bins of CRs. There is a substantial number of events with trajectory length greater than $D \gtrsim 10^{3} \mathrm{Mpc}$ for each energy bin. Thus, the trajectory lengths of CRs are comparable to the mean free paths of pp-interactions and photopion production in the CMB and EBL case, so that these interactions can produce secondary particles including gamma rays and neutrinos.

\subsection{CR Flux Calculation}

To study the propagation of CRs in the diffuse ICM, we used the transport equation as implemented in CRPropa 3 by (Merten et al. 2017, see also equation 1). There are three possible scenarios in CRPropa3 for each particle until its detection: the particle reaches the detector within a Hubble time; the energy of the particle becomes smaller than a given threshold; or the trajectory length of a CR exceeds the maximum propagation distance allowed.

We inject CRs isotropically with a power-law energy distribution with spectral index $\alpha$ and exponential cut-off energy $E_{\max }$ which follows the relation $d N_{\mathrm{CR}, E} / d E \propto E_{i}^{-\alpha} \exp \left(-E_{i} / E_{\max }\right)$ (see Appendix B). We take different values for $\alpha \simeq 1.5-2.7$, and for $E_{\max }=5 \times 10^{15}-10^{18} \mathrm{eV}$ (e.g. Brunetti \& Jones 2014; Fang \& Olinto 2016; Brunetti et al. 2017; Hussain et al. 2019, for review).

As stressed, the lower and upper limits of the mass of the galaxy clusters are taken to be $10^{12} M_{\odot}$ and $10^{16} M_{\odot}$, respectively. This is because for $10^{14} \lesssim E / \mathrm{eV} \lesssim 10^{19}$, clusters with mass $M<10^{12} M_{\odot}$ barely contribute to the total flux of neutrino, due to low gas density, while there are few clusters with $M \gtrsim 10^{15} M_{\odot}$ at high redshifts $(z>1.5)$ (Komatsu et al. 2009; Ade et al. 2014). The closest galaxy clusters are located at $z \sim 0.01$, so we consider the redshift range $0.01 \leq z \leq 5.0$.

The amount of power of the clusters that goes into CR production is left as a free parameter to be regulated by the observations (e.g. Gonzalez et al. 2013; Brunetti \& Jones 2014; Fang \& Olinto 2016, for reviews). We here assume that about $0.5-3 \%$ of the cluster luminosity is available for particle acceleration.

We did not consider the feedback from active galactic nuclei (AGN) or star formation rate (SFR) in our background cosmological simulations (as performed e.g. in Barai et al. 2016; Barai \& de Gouveia Dal Pino 2019). AGN are believed to be the most promising $\mathrm{CR}$ accelerators inside clusters of galaxies and star-forming galax-
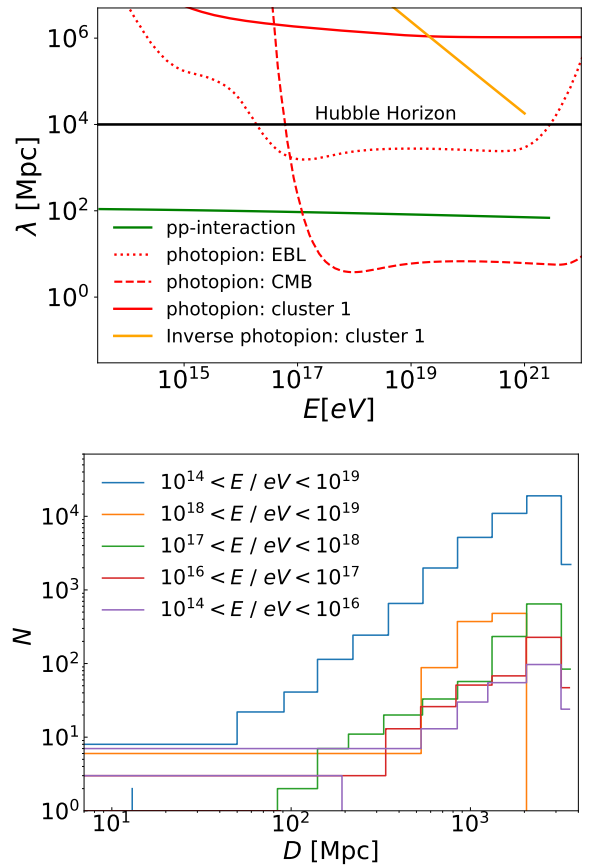

Figure 8. The upper panel shows the mean-free path $\lambda$ for CR interactions which produce neutrinos. It is shown $\lambda$ for photopion production in the bremsstrahung photon field (red solid line), CMB (red dashed line) and EBL (red dotted line). Also shown is $\lambda$ for pp-interactions (green) calculated within a sphere of radius $r=100 \mathrm{kpc}$ around the center of a massive cluster (with mass $10^{15} M_{\odot}$ and shown in Fig. 2). The $\lambda$ for the interaction of high-energy gamma rays with the local gas of the ICM (yellow) is also depicted. The thick black line represents the Hubble horizon in the upper panel. The lower panel shows the distribution of the total trajectory length of CRs inside the cluster as a function of their energy bins.

ies contain many supernova remnants that can also accelerate CRs up to very-high energies ( $E \gtrsim 100 \mathrm{PeV}$ ) (He et al. 2013). AGN are more powerful and more numerous at higher redshifts (Hasinger et al. 2005; Khiali \& de Gouveia Dal Pino 2016; D'Amato et al. 2020), and their luminosity density evolves more strongly for $z \gtrsim 1$. Also, supernovae are more common at high redshifts (He et al. 2013; Moriya et al. 2019). Therefore, it is reasonable to expect that, if high energy cosmic ray (HECR) sources have a cosmological evolution similar to AGN or following the star-formation rate (SFR), then the flux of neutrinos may be higher at high redshifts due to the larger CR output from these objects.

For the evolution of AGN sources (Hopkins \& Beacom 2006; Heinze et al. 2016) and SFR (Yüksel et al. 2008; Wang et al. 2011; Gelmini et al. 2012) we consider the following parametrization:

$$
\begin{aligned}
& \psi_{\mathrm{SFR}}(z)=\frac{1}{B}\left\{\begin{array}{l}
(1+z)^{3.4} \text { if } z<1 \\
(1+z)^{-0.3} \text { if } 1<z<4 \\
(1+z)^{-3.5} \text { if } z>4
\end{array}\right. \\
& \psi_{\mathrm{AGN}}(z)=\frac{(1+z)^{m}}{A}\left\{\begin{array}{l}
(1+z)^{3.44} \text { if } z<0.97 \\
10^{1.09}(1+z)^{-0.26} \text { if } 0.97<z<4.48 \\
10^{6.66}(1+z)^{-7.8} \text { if } z>4.48
\end{array}\right.
\end{aligned}
$$

where $A=360.6$ and $B=6.66$ are normalization constants in equations (5) and (4), respectively. For AGN evolution $\psi_{A G N}(z) \propto$ 
$(1+z)^{5}$, for low redshift $z<1$ (Gelmini et al. 2012; Alves Batista et al. 2019b) and also according to (Gelmini et al. 2012; Heinze et al. 2016), in equation (5), $m>1.5$ for AGN, so we consider $m=1.7$. Typically, the luminosity of AGNs ranges from $10^{42}$ to $10^{47} \mathrm{erg} / \mathrm{s}$ and their evolution depends on their luminosities. The AGNs with luminosities $\sim 10^{44}-10^{46} \mathrm{erg} / \mathrm{s}$ are more important as they are more numerous and believed to be able to accelerate particles to ultra-high energies (e.g. Waxman 2004; Khiali \& de Gouveia Dal Pino 2016). AGNs with luminosities greater than $10^{46} \mathrm{erg} / \mathrm{s}$ are less numerous (Hasinger et al. 2005) and their evolution function $\left(\psi_{\mathrm{AGN}}(z)\right)$ is different from equation (5). For no source evolution, $\psi(z)=1$.

The total flux of CRs is estimated from the entire population of clusters. The number of clusters per mass interval $d N / d M$ at redshift $z$ is given in the lower panel of Fig. 6, which was obtained from our cosmological simulations. It is related to the flux through:

$E^{2} \Phi(E)=\int_{z_{\min }}^{z_{\max }} d z \int_{M_{\min }}^{M_{\max }} d M \frac{d N}{d M} E^{2} \frac{d \dot{N}(E /(1+z), M, z)}{d E}\left(\frac{\psi_{\mathrm{ev}}(z)}{4 \pi d_{L}^{2}(z)}\right)$

where $\psi_{\mathrm{ev}}(z)$ stands for, $\psi_{\mathrm{SFR}}(z)$ and $\psi_{\mathrm{AGN}}(z), \dot{N}$ is the number of CRs per time interval $d t$ with energies between $E$ and $E+d E$ that reaches the observer. The quantity $E^{2} d \dot{N} / d E$ in equation (6) is the power of CRs calculated from our propagation simulation and is several orders of magnitude smaller than the luminosity of observed clusters (e.g., Brunetti \& Jones 2014).

In order to convert the code units of the CR simulation to physical units, we have used a normalization factor (Norm). To calculate Norm, we first evaluate the X-ray luminosity of the cluster using the empirical relation $L_{\mathrm{X}} \propto f_{g}^{2} M_{\mathrm{vir}}$ (Schneider 2014), where $f_{g}=$ $M_{g} / M_{\text {vir }}$ denotes the gas mass $\left(M_{g}\right)$ fraction with respect to the total mass of the cluster within the Virial radius $\left(M_{\mathrm{vir}}\right)$ and then, since we are assuming that $(0.5-3) \%$ of this luminosity goes into CRs, this implies that Norm $\sim(0.5-3) \% L_{\mathrm{X}} / L_{\mathrm{CRsim}}$ and $L_{\mathrm{CRsim}}$ is the luminosity of the simulated CRs. Therefore, the CR power that reaches the observer (at the Earth) is $\sim E^{2} d \dot{N} / d E \times$ Norm. In equation (6) $d_{L}$ is the luminosity distance, given by:

$d_{L}=(1+z) \frac{c}{H_{0}} \int_{0}^{z} \frac{d z^{\prime}}{E\left(z^{\prime}\right)}$,

with

$E(z)=\sqrt{\Omega_{m}(1+z)^{3}+\Omega_{\Lambda}}=\frac{H(z)}{H_{0}}$,

where the Hubble constant, as well as the matter $\left(\Omega_{m}\right)$ and darkenergy $\left(\Omega_{\Lambda}\right)$ densities are defined in section 2.1 , assuming a flat $\Lambda \mathrm{CDM}$ universe.

We selected different injection points inside the clusters of different masses in order to study the spectral dependence with the position, which may correspond to different scenarios of acceleration of CRs. For instance, the larger concentration of galaxies near the center must favor more efficient acceleration, but compressed regions by shocks in the outskirts may also accelerate CRs. The schematic diagram of the simulation of CRs propagation is shown in Fig. 9. CRs are injected at three different positions within each selected cluster denoted by $R_{\text {Offset }}$. The spectra of CRs have been collected by an observer in a sphere of $2 \mathrm{Mpc}$ radius $\left(R_{\mathrm{Obs}}\right)$, centred at the cluster, with a redshift window $(-0.1 \leq z \leq 0.1)$ for all the injection points of CRs. Allflavour neutrino fluxes are also computed at the same observer (see Section-3.4 below).

The spectrum of CRs obtained from our simulations is shown in

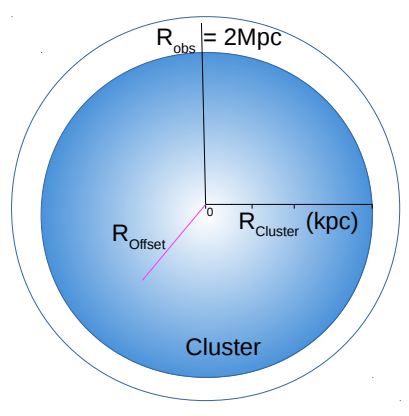

Figure 9. Scheme of the CR simulation geometry. They are injected at three different positions inside each cluster represented by $R_{\mathrm{Offset}}$, and $R_{\mathrm{Obs}}$ is the radius of the observer.

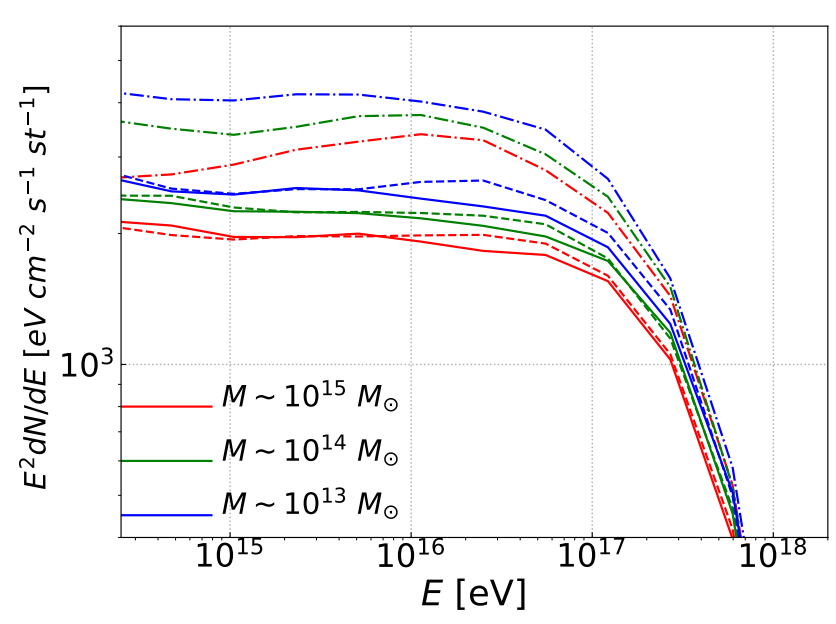

Figure 10. This figure shows the $\mathrm{CR}$ flux of individual clusters of distinct masses, $M \sim 10^{15}$ (red); $10^{14}$ (green); and $M \sim 10^{13} M_{\odot}$ (blue color). This diagram shows the flux of CRs, for sources located at the center of the cluster (solid), at $300 \mathrm{kpc}$ (dashed), and at $1 \mathrm{Mpc}$ (dash-dotted lines) away from the centre. The flux is computed at the edge of the clusters. The spectral parameters are $\alpha=2$ and $E_{\max }=5 \times 10^{17} \mathrm{eV}$, and it is assumed that $2 \%$ of the luminosity of the clusters is converted into CRs.

Figs. $10 \& 11$. Its dependence on the position where the CR source is located within the cluster for $z=0.01$ is shown for three clusters of different masses in Fig. 10. Particles injected at $1 \mathrm{Mpc}$ distance away from the clusters center can leave them in short time, with almost no interaction, as both the magnetic field and the gas number density are very low compared to the central regions. On the other hand, CRs injected at the center or at $300 \mathrm{kpc}$ away from the cluster center can be easily deflected by the magnetic field and trapped in dense regions. This explains the higher CR flux for the injection point at $1 \mathrm{Mpc}$ in Fig. 10. Also, because the confinement of CRs in the central regions of the clusters is comparable to a Hubble time, and because of the value of $\lambda$ for the relevant interactions, the production of secondary particles including neutrinos and gamma rays in the clusters is substantial, as we will see in section 3.4.

In Fig. 11 we show the CR spectrum of all the clusters at different redshifts integrated up to the Earth. Although the spectra in this diagram have been integrated up to the Earth, we have not considered any interactions of the CRs with the background photon and magnetic fields during their propagation from the edge of the clusters 


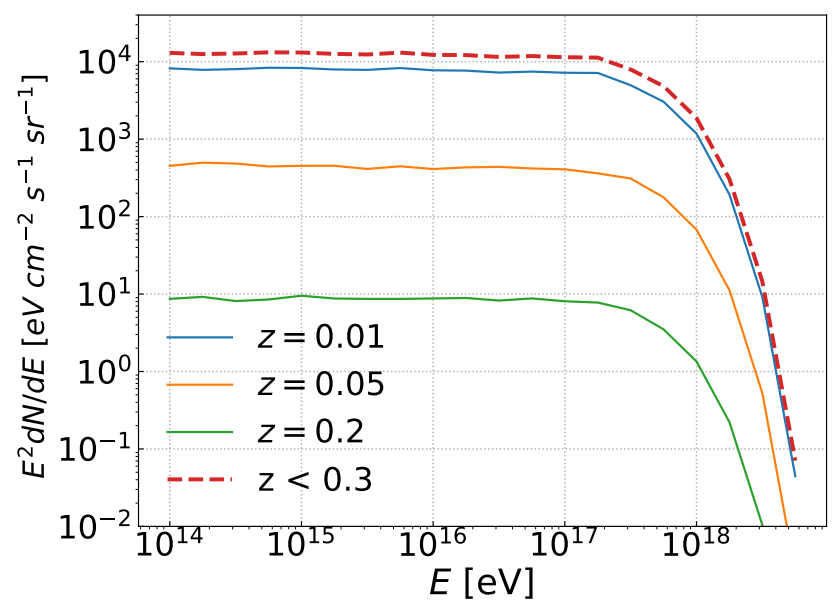

Figure 11. This figure shows the total CR flux (at the Earth distance) from all the clusters distributed in different redshifts: $z=0.01$ (blue); $z=0.05$ (orange); $z=0.2$ (green). The total CRs flux for the redshift range $0.01 \leq$ $z \leq 0.3$ is given by the red dotted line.

to the Earth. Though not quantitatively realistic, it provides important qualitative information. One obvious result is that most of the contribution in the CR flux comes from clusters at low redshifts. Moreover there is a significant suppression in the flux of CRs at $\gtrsim 10^{17} \mathrm{eV}$, which indicates the trapping of lower-energy CRs within the clusters (Alves Batista et al. 2018).

\subsection{Flux of Neutrinos}

To calculate the neutrino flux, the CRPropa 3 code integrates a relation similar to equation (6) for neutrino species, and the procedure is the same as described in Section 3.3 .

In general, neutrino production occurs mainly due to photopion production and pp-interactions. In Fig. 8, where we show $\lambda$ for different interactions, we see that protons with energies $E<10^{17}$ $\mathrm{eV}$ produce neutrinos principally due to pp-interactions, while for $E>10^{17} \mathrm{eV}$, they produce neutrinos both, by pp-interactions and photopion process. We have also seen in Fig. 8 (lower panel) that the total trajectory length of CRs inside a cluster is comparable or larger than $\lambda$ for these interactions and thus, neutrino production is inevitable.

In Fig. 12 we show the dependence of the neutrino flux with the position of the corresponding CR source within clusters of different masses. As in the case of the CR flux, it can be seen that there is less neutrino production for the injection position at $1 \mathrm{Mpc}$ away from the center of the cluster. Furthermore, massive clusters produce more neutrinos than the light ones. In Fig. 13 we present the redshift distribution of neutrinos as a function of their energy, as observed at a distance of $2 \mathrm{Mpc}$ from the center of individual clusters with different masses.

In Fig. $14 \&$ 15, we present the total flux of neutrinos from the whole population of clusters, as measured at Earth, integrated over the entire redshift range within the Hubble time (solid brown curve in the panels). In the left panel of Fig. 14 and in Fig. 15, the injected CR spectrum is assumed to follow $E^{-1.5}$, with an exponential cut-off $E_{\max }=5 \times 10^{16} \mathrm{eV}$. Also, we assumed in these cases that $0.5 \%$ of the kinetic energy of the clusters is converted to the CRs. Besides the total flux, this panel also shows the flux of neutrinos for several

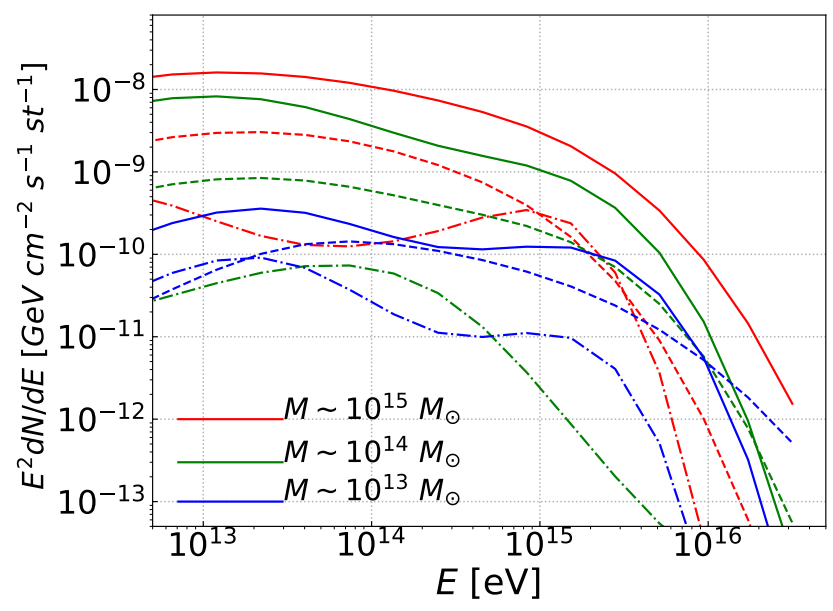

Figure 12. This figure shows the neutrino flux of individual clusters of distinct masses: $M \sim 10^{15}$ (red); $10^{14}$ (green) and $10^{13} M_{\odot}$ (blue color). The CR sources are located at the center of the cluster (solid lines), at $300 \mathrm{kpc}$ (dashed lines), and at $1 \mathrm{Mpc}$ away from the center (dash-dotted lines). The flux is computed at the edge of clusters. The CR injection follows $d N / d E \propto E^{-2}$, $E_{\max }=5 \times 10^{16} \mathrm{eV}$, and it is assumed that $2 \%$ of the luminosity of the clusters is converted to CRs.

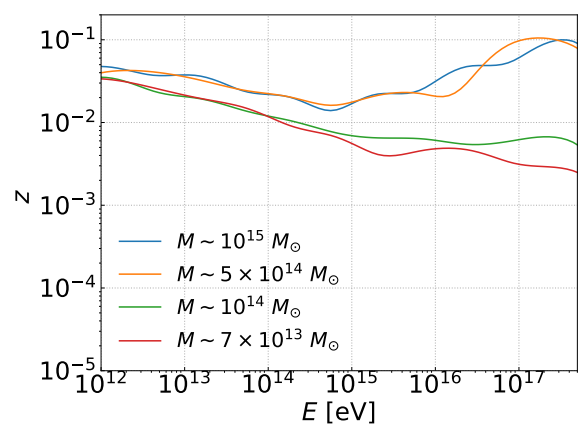

Figure 13. Redshift distribution of the neutrinos as a function of their energy, as observed at $2 \mathrm{Mpc}$ away from the center of clusters with different masses.

cluster mass intervals. The softening effect at higher energies is due to the shorter diffusion time of the CRs, and to the mass distribution of the clusters, as higher flux reflects lower population of massive clusters. In Fig. 15 we present the integrated flux in different redshift intervals and it can also be seen that the clusters at high redshift contribute less to the total flux of neutrinos. Those at $z>1$ barely contribute to the flux due to the low population of massive clusters and their large distances. Fig. 14 and 15 also compares our results with the IceCube observations. We see that for the assumed scenario for CRs injection in left panel of Fig. 14 and in Fig. 15, they can reproduce the IceCube observations for $E>20 \mathrm{TeV}$. In right panel of Fig. 14, instead, we have assumed that $2 \%$ of the kinetic energy of the clusters is converted into CRs, with a CR energy power-law spectrum $E^{-2}$, with $E_{\max }$ following the dependence below with the cluster mass and magnetic field:

$E_{\max }=2.8 \times 10^{18}\left(\frac{M_{\text {cluster }}}{10^{15} M_{\odot}}\right)^{2 / 3}\left(\frac{B_{\text {cluster }} \mathrm{G}}{10^{-6} \mathrm{G}}\right) \mathrm{eV}$,

which is similar to Fang \& Olinto (2016). In this scenario we find that 
the clusters contribution to the neutrino flux is smaller than IceCube measurements.

For all diagrams of Fig. $14 \& 15$, we also compare our results with those of Fang \& Olinto (2016)) (blue lines). The total fluxes in both are similar, in general.Moreover, we see that in both cases, the largest contribution to the flux of neutrinos comes from the cluster mass group $10^{14} M_{\odot}<M<10^{15} M_{\odot}$. However, the contribution from the mass group $10^{12} M_{\odot}<M<10^{14} M_{\odot}$ in our results is a factor twice larger than that of Fang \& Olinto (2016), and smaller by the same factor for the mass group $M>10^{15} M_{\odot}$, at energies $E>0.01 \mathrm{PeV}$ (left panel of Fig. 14).

A striking difference between the two results is that, according to Fang \& Olinto (2016), the redshift range $0.3 \leq z \leq 1$ amounts for the largest contribution to neutrino production, but in our case the redshift range $0.01 \leq z \leq 0.3$ provides a more significant contribution (see Fig. 15). Besides, there is a difference of factor $\sim 2$ to $\sim 3$ between ours and their results at these redshift ranges. This difference may be due to the more simplified modeling of the background distribution of clusters in their case specially for the lower mass group $\left(10^{12} M_{\odot}<M<10^{14} M_{\odot}\right)$ at high redshifts $(z>1)$.

In Fig. 16, we present the total neutrino spectra calculated for different spectral indices of the injected CRs, while in Fig. 17 we show the total neutrino spectra calculated for several cut-off energies. In order to try to fit the observed IceCube data, we have considered a $3 \%$ conversion of the kinetic energy of the cluster into CRs in Figs. $16 \& 17$.

So far, we have computed the $\mathrm{CR}$ and neutrino fluxes from the clusters, considering no evolution function with redshift for both $\mathrm{CR}$ sources, AGN and SFR, i.e. we assumed $\psi_{\mathrm{ev}}(z)=1$ in equation (6). In Fig. 18, we have included these contributions and plotted the flux of neutrinos for the redshift ranges: $z<0.3,0.3<z<1.0$, and $1.0<z<5$.0. The flux is obtained for spectral index $\alpha=2$ and cutoff energy $E_{\max }=5 \times 10^{17} \mathrm{eV}$.

Clusters can directly accelerate CRs through shocks, but any type of astrophysical object that can produce HECRs can also contribute to the diffuse neutrino flux. In the former case, the sources evolve only according to the background MHD simulations, dubbed here "no evolution", whereas in the latter some assumptions have to be made regarding the CR sources. In Fig. 18 we illustrate the impact of the source evolution. We consider, in addition to the case wherein sources do not evolve, SFR and AGN-like evolutions (see equations 5 and 4 and accompanying discussion). Our results suggest that, while the neutrino fluxes for the AGN and the SFR evolutions are relatively close to each other, the case without evolution contributes slightly less to the total flux. Moreover, at high redshifts $(1.0<z<5.0)$, AGNs in clusters produce more neutrinos than sources with SFR-like evolutions, whereas the same is not true for $z \lesssim 1$.

In Fig. 19, we plotted the flux for different combinations of spectral index $\alpha$ and $E_{\max }$, with different source evolution assumptions as in Fig. 18. In both panels all the combinations of $\alpha$ and $E_{\max }$ are roughly matching with IceCube data, except $\alpha=1.5$, and $E_{\text {max }}=5 \times 10^{17} \mathrm{eV}$ in the upper panel as it overshoots the IceCube points.

\section{DISCUSSION}

In our simulations, the central magnetic field strength and gas number density of the ICM are $\sim 10 \mu \mathrm{G}$ and $\sim 10^{-2} \mathrm{~cm}^{-3}$, respectively, for a cluster with mass $10^{15} M_{\odot}$ at $z=0.01$, and both decrease toward the outskirts of the cluster. These quantities depend on the mass of the clusters, being smaller for less massive clusters (see Fig. 2 \& 5). Thus, high-energy CRs will escape with a higher probability without much interactions in the case of less massive clusters. Lower-energy CRs, on the other hand, contribute less to the production of highenergy neutrinos. Therefore, we have a lower neutrino flux from less massive clusters. In contrast, for massive clusters, higher magnetic field and gas density produce higher neutrino flux due to the longer confinement time, as we see in Fig. 12.

We tested several injection CRs spectral indices $(\alpha \simeq 1.5-2.7)$, cut-off energies $\left(E_{\max }=5 \times 10^{15}-10^{18} \mathrm{eV}\right)$, and source evolution (AGN, SFR, no evolution), in order to try to interpret the IceCube data (see Figs. 14, 15, 16, 17, 18 and 19). Overall, our results indicate that galaxy clusters can contribute to a considerable fraction of the diffuse neutrino flux measured by IceCube at energies between $100 \mathrm{TeV}$ and $10 \mathrm{PeV}$, or even all of it, provided that that protons compose most of the CRs.

Our results also look, in principle, similar to those of Fang \& Olinto (2016) with no source evolution, who considered essentially the same redshift interval, but employed semi-analytical profiles to describe the cluster properties. In particular, in both cases, the largest contribution to the flux of neutrinos comes from the cluster mass group $10^{14}<M<10^{15} M_{\odot}$. However, they did not consider the interactions of CRs with CMB and EBL background as they considered it subdominant compared to the hadronic background following Kotera et al. (2009). But, it can be seen from the upper panel of Fig. 8 that $\lambda$ for pp-interaction and photopion production in the CMB are comparable for CRs of energy $\gtrsim 10^{17} \mathrm{eV}$. Therefore, the neutrino production due to $\mathrm{CR}$ interactions with the $\mathrm{CMB}$ is not negligible. Perhaps the most relevant difference between our results and theirs is that, in their case, the redshift range $0.3 \leq z \leq 1$ makes the largest contribution to neutrino production, while in our case this comes from the redshift range $z \lesssim 0.3$, when considering no source evolution (see Fig 15).

When including source evolution, there is also a dominance in the neutrino flux from the redshift range $z \lesssim 0.3$, though the contribution due to the evolution of star forming galaxies (SFR) from redshifts $0.3 \leq z \leq 1$ is also important. Overall, the inclusion of source evolution can increase the diffuse neutrino flux by a factor of $\sim 3$ (when considering the separate contributions of AGN or SFR) to $\sim 5$ (when considering both contributions concomitantly) in the cases we studied, compared to the case with no evolution, which is in agreement with (Murase \& Waxman 2016). Also, our results agree with the IceCube measurements for $E \gtrsim 10^{14} \mathrm{eV}$ and are in rough accordance with (Murase 2017; Fang \& Murase 2018). Nevertheless, since there are uncertainties related to the choice of specific populations for the $\mathrm{CR}$ sources, obtaining a full picture of the diffuse high-energy neutrino emission by clusters is not a straightforward task.

It is also worth comparing our results with Zandanel et al. (2015), who evaluated the neutrino spectrum based on estimations of the radio to gamma-ray luminosities of the clusters in the universe. Although our work has assumed an entirely different approach, both results are consistent, especially for a CR spectral index $\alpha \simeq 2$. High-energy ( $E>10^{17} \mathrm{eV}$ ) CRs can escape easily from clusters, effectively leading to a spectral steepening that was not considered by Zandanel et al. (2015). However, not all the clusters are expected to produce hadronic emission (Zandanel et al. 2015, 2014). In fact, we observe less hadronic interactions in the case of low-mass clusters $\left(M \lesssim 10^{14} M_{\odot}\right)$, which could further limit the neutrino contribution from clusters.

The cluster scenario may get strong backing due to anisotropy detections above PeV energies. Recently, only a few sources of highenergy neutrinos have been observed (Aartsen et al. 2013, 2015; Albert et al. 2018; Ansoldi et al. 2018; Aartsen et al. 2020), but 

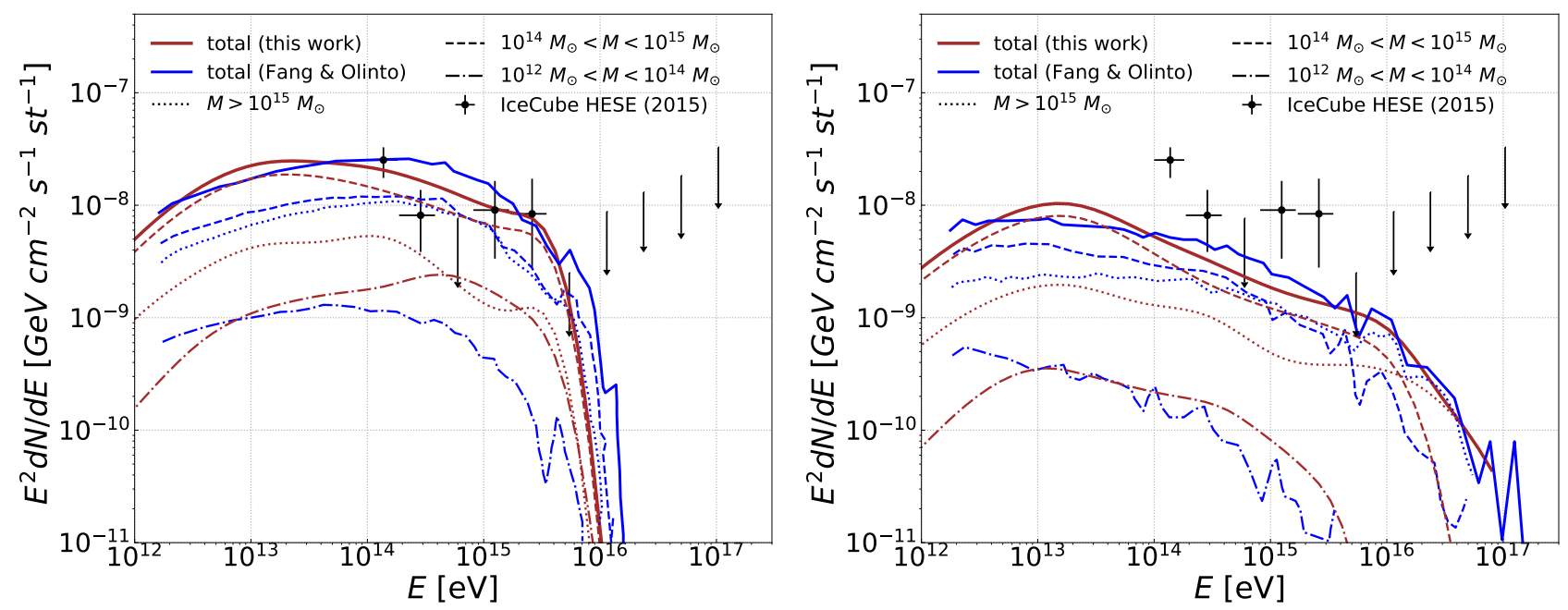

Figure 14. Neutrino spectrum at Earth obtained using our simulations (brown lines), compared with the IceCube data (markers), and Fang \& Olinto (2016) results (blue lines). The panels show the total flux integrated over all clusters and redshifts between $0.01 \leq z \leq 5$ (solid thick lines). The left panel shows the neutrino spectra (thin blue and brown lines) for cluster mass ranges of: $10^{12} M_{\odot}<M<10^{14} M_{\odot}$ (dash-dotted), $10^{14} M_{\odot}<M<10^{15} M_{\odot}$ (dashed), and $M>10^{15} M_{\odot}$ (dotted lines). The left panel corresponds to the case with $\alpha=1.5$ and $E_{\max }=5 \times 10^{16} \mathrm{eV}$, whereas in the right panel $\alpha=-2$ and $E_{\max }$ follows equation (9). These diagrams do not include the redshift evolution of the CR sources, $\psi_{e v}=1$ in equation equation (6).

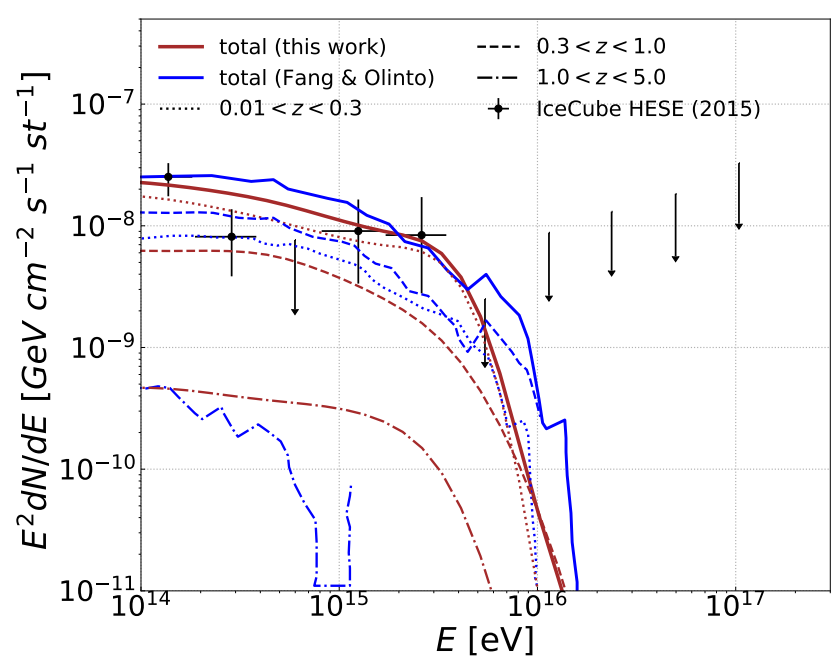

Figure 15. This figure shows the neutrino spectrum for different redshift ranges: $z<0.3$ (dotted lines), $0.3<z<1.0$ (dashed), and $1.0<z<5.0$ (dash-dotted lines). The solid blue and brown lines correspond to the total spectrum in Fang \& Olinto (2016), and in this work, respectively. The CR injection in this figure follows $d N / d E \propto E^{-1.5}$, and $E_{\max }=5 \times 10^{16} \mathrm{eV}$. This figure does not include the redshift evolution of the CR sources, $\psi_{e v}=1$ in equation equation (6).

there are also expectations to increase the observations with future instruments like IceCube-Gen2 (The IceCube-Gen2 Collaboration 2020), KM3NeT (Adrián-Martínez et al. 2016), and the Giant Radio Array for Neutrino Detection (GRAND) (Álvarez-Muñiz et al. 2020). Specifically, neutrinos from clusters are more likely to be observed if the flux of cosmogenic neutrinos is low, which might contaminate the signal, as discussed by Alves Batista et al. (2019b).

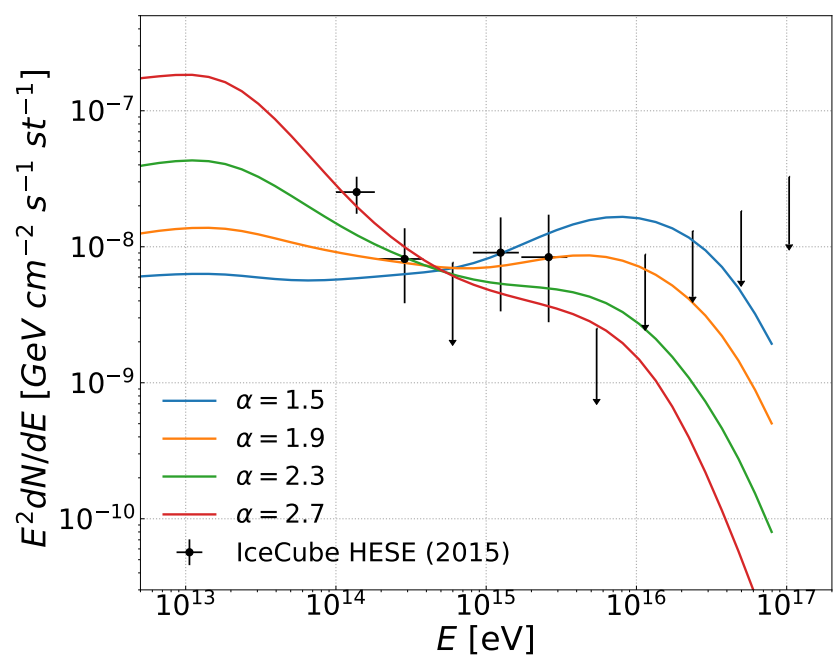

Figure 16. Total spectrum of neutrinos for different injected CR spectra, $\sim E^{-\alpha}$, with $\alpha=1.5$ (blue), 1.9 (orange), 2.3 (green), 2.7 (red). We consider $E_{\max }=5 \times 10^{17} \mathrm{eV}$. This figure does not include the redshift evolution of the CR sources, $\psi_{e v}=1$ in equation equation (6).

\section{CONCLUSIONS}

We considered a cosmological background based on 3D-MHD simulations to model the cluster population of the entire universe, and a multidimensional Monte Carlo technique to study the propagation of CRs in this environment and obtain the flux of neutrinos they produce. Our results can be summarized as follows:

- We found that CRs with energy $E \lesssim 10^{17} \mathrm{eV}$ cannot escape from the innermost regions of the clusters, due to interactions with the background gas, thermal photons and magnetic fields. Massive clusters $\left(M \gtrsim 10^{14} M_{\odot}\right)$ have stronger magnetic fields which can 

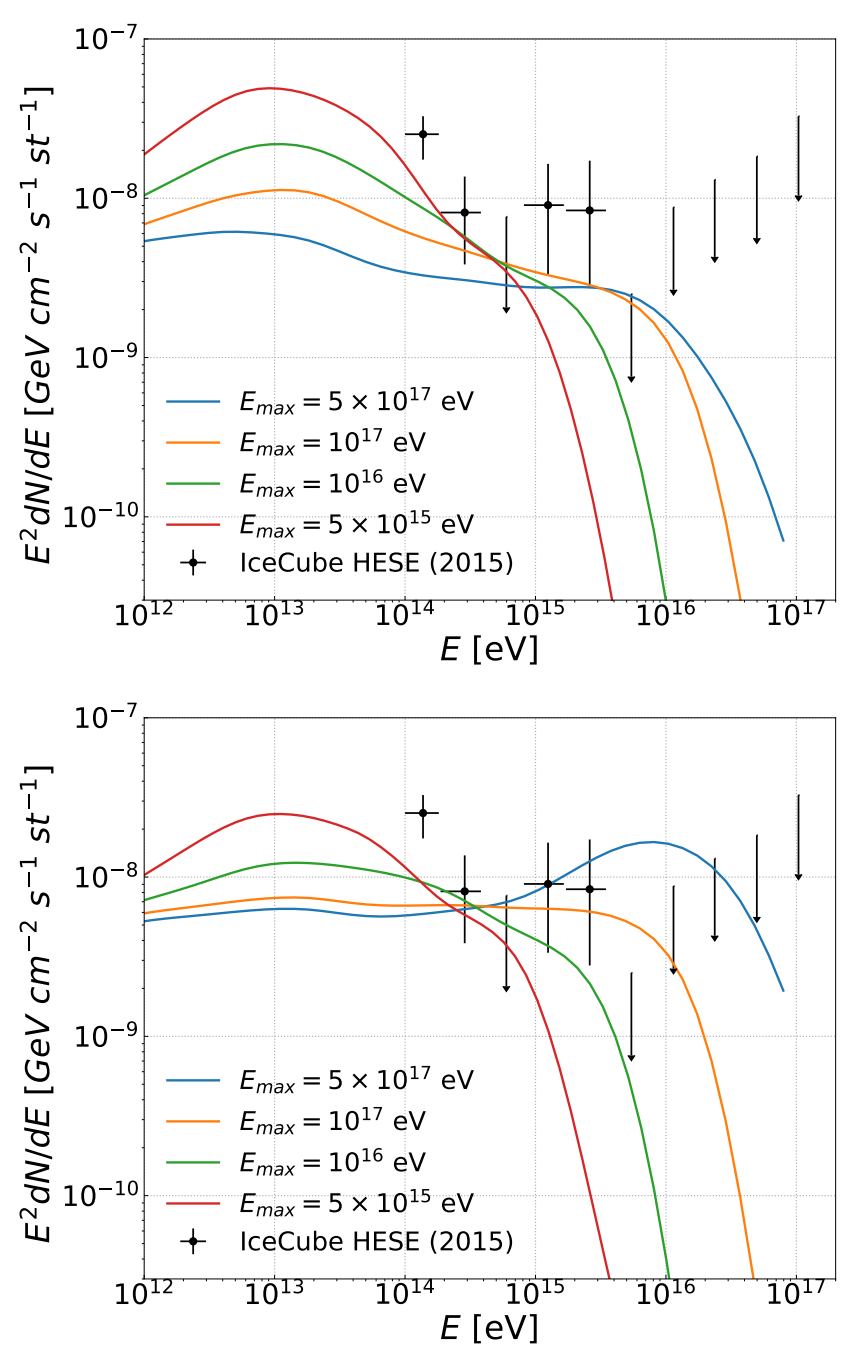

Figure 17. Total neutrino spectrum for different cutoff energies i.e., $E_{\text {max }}=$ $5 \times 10^{15}$ (red), $10^{16}$ (green), $10^{17}$ (orange), and $5 \times 10^{17} \mathrm{eV}$ (blue). In the upper panel the spectral index is $\alpha=2$, and in lower panel $\alpha=1.5$. This figure does not include the redshift evolution of the CR sources, $\psi_{e v}=1$ in equation equation (6).

confine these high-energy CRs for a time comparable to the age of the universe.

- Our simulations predict that the neutrino flux above PeV energies comes from the most massive clusters because the CR interactions with the gas of the ICM are rare for clusters with $M<10^{14} M_{\odot}$.

- Most of the neutrino flux comes from nearby clusters in the redshift range $z \lesssim 0.3$. The high-redshif clusters contribute less to the total flux of neutrinos compared to the low-redshift ones, as the population of massive clusters at high redshifts is low.

- The total integrated neutrino flux obtained from the interactions of CRs with the ICM gas and CMB during their propagation in the turbulent magnetic field can account for sizeable percentage of the IceCube observations, especially, between energy $100 \mathrm{TeV}$ and $10 \mathrm{PeV}$.

- Our results also indicate that the redshift evolution of CR sources like AGN and SFR, enhance the flux of neutrinos.

Finally, more realistic studies considering cosmological simulations that account for AGN and star formation feedback from galax-

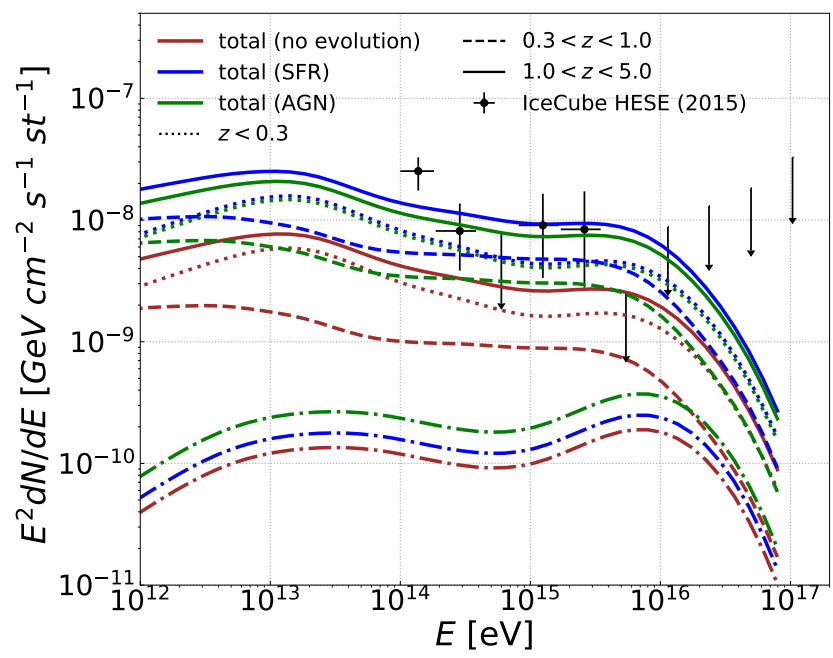

Figure 18. Neutrino spectrum for different assumptions on the evolution of the CR sources: SFR (blue), AGN (green), and no evolution (brown). The fluxes are shown for different redshift ranges: $z<0.3$ (dotted lines), $0.3<z<1.0$ (dashed), and $1.0<z<5.0$ (dash-dotted lines). The CR injection spectrum has parameters $\alpha=2$ and $E_{\max }=5 \times 10^{17} \mathrm{eV}$.

ies ( e.g. Barai et al. 2016; Barai \& de Gouveia Dal Pino 2019) will allow to constrain better the redshift evolution of the CR sources in the computation of the total neutrino flux from clusters. Furthermore, in the future, IceCube will have detected more events. Then, combined with diffuse gamma-ray searches by the forthcoming CTA (Cherenkov Telescope Array Consortium et al. 2019), it will be possible to better assess the contribution of galaxy clusters to the total extragalactic neutrino flux.

\section{ACKNOWLEDGEMENTS}

Saqib Hussain acknowledges support from the Brazilian funding agency CNPq. EMdGDP is also grateful for the support of the Brazilian agencies FAPESP (grant 2013/10559-5) and CNPq (grant $308643 / 2017-8$ ). RAB is currently funded by the Radboud Excellence Initiative, and received support from FAPESP in the early stages of this work (grant 17/12828-4). KD acknowledges support by the Deutsche Forschungsgemeinschaft (DFG, German Research Foundation) under Germany's Excellence Strategy - EXC-2094 - 39078331 and by the funding for the COMPLEX project from the European Research Council (ERC) under the European Union's Horizon 2020 research and innovation program grant agreement ERC-2019-AdG 860744. The numerical simulations presented here were performed in the cluster of the Group of Plasmas and High-Energy Astrophysics (GAPAE), acquired with support from FAPESP (grant 2013/105595). This work also made use of the computing facilities of the Laboratory of Astroinformatics (IAG/USP, NAT/Unicsul), whose purchase was also made possible by a FAPESP (grant 2009/54006-4). We also acknowledge very useful comments from K. Murase on an earlier version of this manuscript.

\section{REFERENCES}

Aab A., et al., 2018, The Astrophysical Journal, 868, 4

Aartsen M. G., et al., 2013, Physical review letters, 111, 021103 

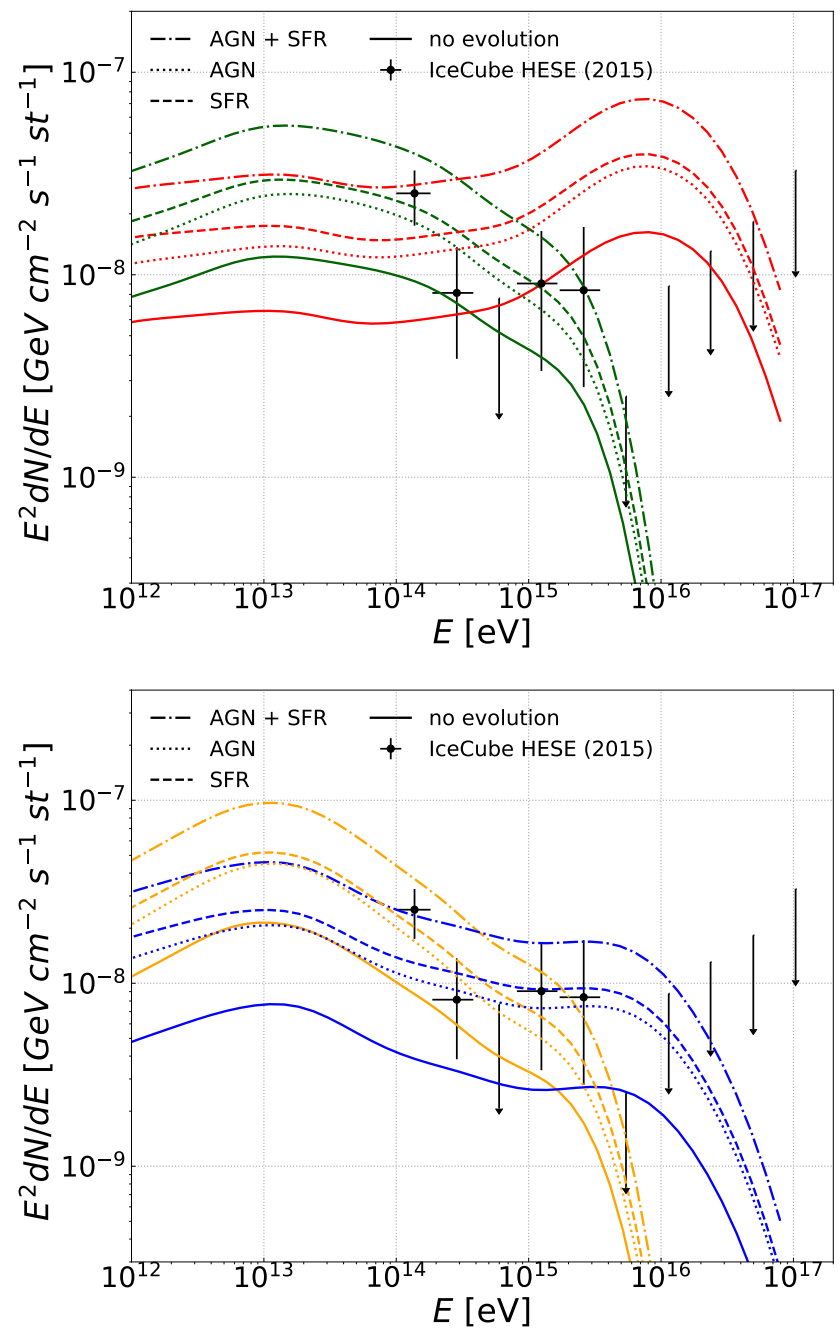

Figure 19. Flux of neutrinos for different assumptions on the evolution of the CR sources: no evolution (solid lines), SFR (dashed lines), AGN (dotted lines) and AGN + SFR (dash-dotted lines). In upper panel green and red lines represent $\alpha=1.5$ for $E_{\max }=10^{16}$ and $5 \times 10^{17} \mathrm{eV}$ respectively. In lower panel orange and blue lines correspond to $\alpha=2$ for $E_{\max }=10^{16}$ and $5 \times 10^{17} \mathrm{eV}$, respectively.

Aartsen M., et al., 2015, Physical Review D, 91, 022001

Aartsen M., et al., 2017, The Astrophysical Journal, 835, 45

Aartsen M., et al., 2020, Physical review letters, 124, 051103

Ackermann M., et al., 2019, arXiv preprint arXiv:1903.04334

Ade P. A., et al., 2014, Astronomy \& Astrophysics, 571, A16

Adrián-Martínez S., et al., 2016, Journal of Physics G Nuclear Physics, 43, 084001

Ahlers M., Halzen F., 2018, Progress in Particle and Nuclear Physics, 102, 73

Albert A., et al., 2018, The Astrophysical Journal Letters, 863, L30

Aloisio R., Berezinsky V., Gazizov A., 2012, Astroparticle Physics, 39, 129

Álvarez-Muñiz J., et al., 2020, Science China Physics, Mechanics \& Astronomy, 63, 219501

Alves Batista R., et al., 2016, Journal of Cosmology and Astroparticle Physics, 2016, 038

Alves Batista R., Pino E., Dolag K., Hussain S., 2018, arXiv preprint arXiv: 1811.03062

Alves Batista R., et al., 2019a, Frontiers in Astronomy and Space Sciences, 6,23
Alves Batista R., de Almeida R. M., Lago B., Kotera K., 2019b, Journal of Cosmology and Astroparticle Physics, 2019, 002

Amato E., Blasi P., 2018, Advances in Space Research, 62, 2731

Anchordoqui L. A., Paul T. C., da Silva L. H., Torres D. F., Vlcek B. J., 2014, Physical Review D, 89, 127304

Ansoldi S., et al., 2018, The Astrophysical Journal Letters, 863, L10

Apel W., et al., 2013, Astroparticle Physics, 47, 54

Barai P., de Gouveia Dal Pino E. M., 2019, MNRAS, 487, 5549

Barai P., Murante G., Borgani S., Gaspari M., Granato G. L., Monaco P., Ragone-Figueroa C., 2016, MNRAS, 461, 1548

Berezinsky V. S., Blasi P., Ptuskin V., 1997, The Astrophysical Journal, 487, 529

Blasi P., 2013, The Astronomy and Astrophysics Review, 21, 70

Brunetti G., Jones T. W., 2014, International Journal of Modern Physics D, 23, 1430007

Brunetti G., Vazza F., 2020, Physical Review Letters, 124, 051101

Brunetti G., Zimmer S., Zandanel F., 2017, Monthly Notices of the Royal Astronomical Society, 472, 1506

Chakraborty S., Izaguirre I., 2015, Physics Letters B, 745, 35

Cherenkov Telescope Array Consortium et al., 2019, Science with the Cherenkov Telescope Array, doi:10.1142/10986.

Dolag K., Grasso D., Springel V., Tkachev I., 2005, Journal of Cosmology and Astroparticle Physics, 2005, 009

Dominguez A., et al., 2011, Monthly Notices of the Royal Astronomical Society, 410, 2556

D’Amato Q., et al., 2020, Astronomy \& Astrophysics, 636, A37

Fang K., Murase K., 2018, Nature Physics, 14, 396

Fang K., Olinto A. V., 2016, The Astrophysical Journal, 828, 37

Ferrari C., Govoni F., Schindler S., Bykov A., Rephaeli Y., 2008, in , Clusters of Galaxies. Springer, pp 93-118

Gelmini G. B., Kalashev O., Semikoz D. V., 2012, Journal of Cosmology and Astroparticle Physics, 2012, 044

Giacinti G., Kachelrieß M., Semikoz D., 2015, Physical Review D, 91, 083009

Gilmore R., Somerville R., Primack J., Domínguez A., 2012, Not. Roy. Astron. Soc, 422,1104

Gonzalez A. H., Sivanandam S., Zabludoff A. I., Zaritsky D., 2013, The Astrophysical Journal, 778, 14

Gouin C., Aghanim N., Bonjean V., Douspis M., 2020, Astronomy \& Astrophysics, 635, A195

Govoni F., et al., 2019, Science, 364, 981

Hasinger G., Miyaji T., Schmidt M., 2005, Astronomy \& Astrophysics, 441, 417

He H.-N., Wang T., Fan Y.-Z., Liu S.-M., Wei D.-M., 2013, Physical Review D, 87, 063011

Heinze J., Boncioli D., Bustamante M., Winter W., 2016, The Astrophysical Journal, 825, 122

Hopkins A. M., Beacom J. F., 2006, The Astrophysical Journal, 651, 142

Hümmer S., Baerwald P., Winter W., 2012, Physical Review Letters, 108, 231101

Hussain S., Alves Batista R., Dal Pino E. M. d. G., 2019, in ICRC. p. 81

Kachelriess M., 2019, in EPJ Web of Conferences. p. 04003

Kafexhiu E., Aharonian F., Taylor A. M., Vila G. S., 2014, Physical Review D, 90, 123014

Kashiyama K., Mészáros P., 2014, The Astrophysical Journal Letters, 790, L14

Khiali B., de Gouveia Dal Pino E. M., 2016, MNRAS, 455, 838

Kim J., Ryu D., Kang H., Kim S., Rey S.-C., 2019, Science advances, 5, eaau8227

Komatsu E., et al., 2009, The Astrophysical Journal Supplement Series, 180, 330

Kotera K., Allard D., Murase K., Aoi J., Dubois Y., Pierog T., Nagataki S., 2009, The Astrophysical Journal, 707, 370

Liu R.-Y., Wang X.-Y., 2013, The Astrophysical Journal, 766, 73

Merten L., Tjus J. B., Fichtner H., Eichmann B., Sigl G., 2017, Journal of Cosmology and Astroparticle Physics, 2017, 046

Moriya T. J., et al., 2019, The Astrophysical Journal Supplement Series, 241, 16 
Murase K., 2017, in , neutrino astronomy: current status, future prospects. World Scientific, pp 15-31

Murase K., Waxman E., 2016, Physical Review D, 94, 103006

Murase K., Inoue S., Nagataki S., 2008, The Astrophysical Journal Letters, 689, L105

Murase K., Ahlers M., Lacki B. C., 2013, Physical Review D, 88, 121301

Parizot E., 2014, arXiv preprint arXiv:1410.2655

Rodríguez-Ramírez J. C., de Gouveia Dal Pino E. M., Alves Batista R., 2019, ApJ, 879, 6

Rordorf C., Grasso D., Dolag K., 2004, Astroparticle Physics, 22, 167

Rybicki G. B., Lightman A. P., 2008, Radiative processes in astrophysics. John Wiley \& Sons

Schlickeiser R., 2002, in , Cosmic Ray Astrophysics. Springer, pp 383-389

Schneider P., 2014, Extragalactic astronomy and cosmology: an introduction. Springer

Senno N., Mészáros P., Murase K., Baerwald P., Rees M. J., 2015, The Astrophysical Journal, 806, 24

Springel V., 2005, Monthly notices of the royal astronomical society, 364, 1105

Springel V., Yoshida N., White S. D., 2001, New Astronomy, 6, 79

The IceCube-Gen2 Collaboration 2020, arXiv e-prints, p. arXiv:2008.04323

Thoudam S., Rachen J., van Vliet A., Achterberg A., Buitink S., Falcke H., Hörandel J., 2016, Astronomy \& Astrophysics, 595, A33

Voit G. M., 2005, Reviews of Modern Physics, 77, 207

Wang X.-Y., Liu R.-Y., Aharonian F., 2011, The Astrophysical Journal, 736, 112

Waxman E., 2004, New Journal of Physics, 6, 140

Wolfe B., Melia F., 2008, The Astrophysical Journal, 675, 156

Xu H., Li H., Collins D. C., Li S., Norman M. L., 2009, The Astrophysical Journal Letters, 698, L14

Yoast-Hull T. M., Everett J. E., Gallagher III J., Zweibel E. G., 2013, The Astrophysical Journal, 768, 53

Yüksel H., Kistler M. D., Beacom J. F., Hopkins A. M., 2008, The Astrophysical Journal Letters, 683, L5

Zandanel F., Pfrommer C., Prada F., 2014, Monthly Notices of the Royal Astronomical Society, 438, 124

Zandanel F., Tamborra I., Gabici S., Ando S., 2015, Astronomy \& Astrophysics, 578, A32

\section{APPENDIX A: MEAN FREE PATHS}

The mean free path $\lambda$ for different CR interactions in the ICM are defined below.

For a CR proton with Lorentz factor $\gamma_{p}$ traversing an isotropic photon field, one obtains the rate $\lambda_{p \gamma}^{-1}\left(E_{p}\right)$ (Schlickeiser 2002)

$$
\begin{aligned}
\lambda_{p \gamma}^{-1}\left(E_{p}\right) & =\frac{1}{2 \gamma_{p}} \int_{\epsilon_{\mathrm{th}} / 2 \gamma_{p}}^{\infty} d \epsilon \frac{n_{\mathrm{ph}}\left(\epsilon, r_{i}\right)}{\epsilon^{2}} \int_{\epsilon_{\mathrm{th}}}^{2 \gamma_{p} \epsilon} d \epsilon^{\prime} \epsilon^{\prime} \sigma_{p \gamma}\left(\epsilon^{\prime}\right) K_{p}\left(\epsilon^{\prime}\right) \\
\epsilon_{\mathrm{th}} & =K m_{\pi} c^{2}\left[1+\frac{K m_{\pi}}{2 m_{p}}\right]=145 \mathrm{MeV}
\end{aligned}
$$

Where $n_{\mathrm{ph}}\left(\epsilon, r_{i}\right)$ denotes the number density of photons of energy $\epsilon$ at a given distance $r_{i}$ from the center of the cluster and $\sigma_{p \gamma}$ is the cross section of the interaction of CRs with background photons. The threshold energy for the production of $K$ pions is given by equation (A2), so that for the production of a single $(K=1)$ pion the rest system threshold energy is $\epsilon_{\mathrm{th}}=145 \mathrm{MeV}$ (Schlickeiser 2002).

To calculate the rate for the interactions of high-energy photons (produced during the propagation of CRs inside a cluster) with the local protons in the ICM, we can use equation (A1) with the following modification in the center-of-mass (CM) energy. The energy $E$ and 3 -momentum $\mathbf{p}$ of a particle of mass $m$ form a 4-vector $p=(E, p)$ whose square $p^{2}=(E / c)^{2}-\mathbf{p}^{2}=m^{2} c^{4}$. The velocity of the particle is $\beta c=\mathbf{v} / c=\mathbf{p} / E$. In the collision of two particles of masses $m_{1}$ and $m_{2}$, the total CM energy can be expressed in the Lorentz-invariant form as

$$
\begin{aligned}
\epsilon_{C M} & =\left[\frac{1}{c^{2}}\left(E_{1}+E_{2}\right)^{2}-\left(\mathbf{p}_{\mathbf{1}}+\mathbf{p}_{2}\right) c^{2}\right]^{1 / 2} \\
& =\left[m_{1}^{2} c^{4}+m_{2}^{2} c^{4}+\frac{2 E_{1} E_{2}}{c^{2}}\left(1-\beta_{1} \beta_{2} \cos \theta\right)\right]^{1 / 2},
\end{aligned}
$$

where $\theta$ is the angle between the particles that we can consider zero. In the frame where one particle (of mass $m_{2}$ ) is at rest (lab frame) then,

$\epsilon_{C M}=\left(m_{1}^{2} c^{4}+m_{2}^{2} c^{4}+2 E_{1} m_{2} c^{2}\right)^{1 / 2}$.

If we consider $m_{2}$ is proton and $m_{1}$ is photon, then the above relation becomes

$$
\epsilon_{C M}=\left(m_{2}^{2} c^{4}+2 E_{1} m_{2} c^{2}\right)^{1 / 2} .
$$

$$
\lambda_{\gamma p}^{-1}\left(\epsilon_{\mathrm{ph}}\right)=\frac{\epsilon_{p}}{2 \epsilon_{\mathrm{ph}}} \int_{\epsilon_{\mathrm{th}} /\left(2 \epsilon_{\mathrm{ph}} / \epsilon_{p}\right)}^{\infty} d \epsilon \frac{n_{p}\left(\epsilon, r_{i}\right)}{\epsilon_{p}^{2}} \int_{\epsilon_{\mathrm{th}}}^{\left(2 \epsilon_{\mathrm{ph}} / \epsilon_{p}\right) \epsilon} d \epsilon^{\prime} \epsilon^{\prime} \sigma_{\gamma p}\left(\epsilon^{\prime}\right),
$$

so that the rest frame is in the local protons. We used equation (A6) for the energy of the CM in equation (A7). In equation (A7), $n_{p}\left(\epsilon, r_{i}\right)$ is number density of local protons with energy $\epsilon_{p}=m_{p} c^{2} \sim 1 \mathrm{GeV}$ at a given distance $r_{i}$ from the center of a cluster and decreases toward the outskirt, $\epsilon_{\mathrm{th}} \sim 1.4 \times 10^{8} \mathrm{eV}$ is the threshold energy for this interaction and the cross section $\sigma_{\gamma p}\left(\epsilon^{\prime}\right)$ is of the order $\sim 10^{-37}\left(\mathrm{~cm}^{2}\right)$. With these values used in equation (A7) we solve this integral to calculate $\lambda$ for $\gamma$-proton interaction. We calculated $\lambda$ from equations A1-A7 with some modifications to include the information of the spatially dependent Bremsstrahlung photon field of the clusters $n_{\mathrm{ph}}(\epsilon, r)$.

For proton-proton $(\mathrm{pp})$ interaction, the rate is given by

$\lambda_{\mathrm{pp}}^{-1}\left(E_{p}, r_{i}\right)=K_{\mathrm{pp}} \sigma_{\mathrm{pp}}\left(E_{p}\right) n_{i}\left(r_{i}\right)$

Where $K_{\mathrm{pp}}=0.5$ is the inelasticity factor, $n_{i}\left(r_{i}\right)$ denotes the number density of proton at a given distance $r_{i}$ from the center of the cluster and $E_{p}$ is the energy of the protons.

To obtain the proton number density, we consider that the background plasma consists of electrons and protons in near balancing. Since the abundance is mostly of $\mathrm{H}$ and this is mostly ionized in the hot ICM, this is a reasonable assumption. Thus $n_{p} \simeq n_{e}$, and $\rho_{\text {gas }}=n_{p} m_{p}+n_{e} m_{e} \sim n_{p} m_{p}$, so that $n_{i} \simeq n_{e} \simeq \rho_{\text {gas }} / m_{p}$, where $m_{p}$ is the proton mass and $\rho_{\text {gas }}$ is the gas mass density in the system.

For $\sigma_{\mathrm{pp}}=70 \mathrm{mb}\left(1 \mathrm{barn}=10^{-29} \mathrm{~m}^{2}\right)$, we have for the cross section (Kafexhiu et al. 2014):

$$
\sigma_{\mathrm{pp}}=\left[30.7-0.96 \log \left(\frac{E_{p}}{E_{p}^{\mathrm{th}}}\right)+0.18 \log \left(\frac{E_{p}}{E_{p}^{\mathrm{th}}}\right)\right]\left[1-\left(\frac{E_{p}^{\mathrm{th}}}{E_{p}}\right)^{1.9}\right]^{3} \mathrm{mb},
$$

where $E_{p}$ is the energy of the proton and $E_{p}^{\text {th }}$ is the threshold kinetic energy $E_{p}^{\text {th }}=2 m_{\pi}+m_{\pi} / m_{p} \approx 0.2797 \mathrm{GeV}$. We used equations A8 and (A9) to calculate $\lambda_{\mathrm{pp}}$. 


\section{APPENDIX B: SPECTRAL INDEX}

To calculate the flux of neutrinos corresponding to injected CRs with an arbitrary power-law spectrum with power law index $\alpha$, $d N_{\mathrm{CR}, E} / d E \propto E_{i}^{-\alpha} \exp \left\{-E_{i} / E_{\max }\right\}$, we can normalize the spectrum as follows:

$$
J(\alpha)=\frac{\ln \left(E_{\mathrm{CR}, \max } / E_{\min }\right)}{\int_{E_{\min }, \max }^{E_{\mathrm{m}}} E_{i}^{1-\alpha} \exp \left(-\frac{E_{i}}{E_{\max }}\right) d E} E_{i}^{1-\alpha} \exp \left(-\frac{E_{i}}{E_{\max }}\right)
$$

Where, $E_{i}$ is the injection energy of the simulated CRs, $E_{\max }$ is the exponential cut-off energy, and $E_{\mathrm{CR} \text {, max }}$ is the maximum injection energy of the CRs. 